Final Technical Report

Grant NAG3 - 781

NONLINGAR INTERACTION BETWEEN A PAIR OF OBLIQUE MODES IN A SUPERSONIC MIXING LAYER: IONG-WAVE IIMIT

\author{
THOMAS $F$. BALSA \\ Principal Investigator \\ Department of Aerospace \& Mechanical Engineering \\ University of Arizona \\ Tucson, AZ 85721 \\ JAMES GARTSIDE \\ Prograrn in Applied Mathematics \\ Uridrersity of Arizona \\ Tucson, AZ 8572j.
}

EIIJAI, REPORT

February 1995

PREPARED FOR

NASA Lewis Research Center

Cleveland, OH 44135

UNDER GRANT

NEG3 - 781

(NASA-CR-197380) NONLINEAR

N95-22173

INTERACTION BETHEEN A PAIR OF OBLIQUE MODES IN A SUPERSONIC MIXING LAYER: LONG-WAVE LIMIT Final

Unclas

Technical Report (Arizona Univ.)

$61 \mathrm{p}$

$63 / 340042020$ 


\title{
Nonlinear interaction between a pair of oblique modes in a supersonic mixing layer: long-wave limit
}

\author{
By THOMAS F. BALSA' AND JAMES GARTSIDE² \\ ${ }^{1}$ Department of Aerospace and Mechanical Engineering, University of Arizona. \\ Tucson, AZ 85721, USA \\ 2 Program in Applied Mathematics, University of Arizona, \\ Tucson, AZ 85721, USA
}

The nonlinear interaction between a pair of symmetric, oblique, and spatial instability modes is studied in the long-wave limit using asymptotic methods. The base flow is taken to be a supersonic mixing layer whose Mach number is such that the corresponding vortex sheet is marginally stable according to Miles' criterion. It is shown that the amplitude of the mode obeys a nonlinear integro-differential equation. Numerical solutions of this equation show that, when the obliqueness angle is less than $\pi / 4$, the effect of the nonlinearity is to enhance the growth rate of the instability. The solution terminates in a singularity at a finite streamwise location. This result is reminiscent of that obtained in the vicinity of the neutral point by other authors in several different types of flows. On the other hand, when the obliqueness angle is more than $\pi / 4$, the streamwise development of the amplitude is characterized by a series of modulations. This arises from the fact that the nonlinear term in the amplitude equation may be either stabilizing or destabilizing, depending on the value of the streamwise coordinate. However, even in this case the amplitude of the disturbance increases. though not as rapidly as in the case for which the angle is less than $\pi / 4$. Quite generally then, the nonlinear interaction between two oblique modes in a supersonic mixing layer enhances the growth of the disturbance. 


\section{Introduction}

Recent interest in the linear and nonlinear stability of supersonic boundary layers and free-shear flows has been sparked by the occurrence of these flows in the external and internal aerodynamics of high-speed airplanes and their propulsion systems. While the subject of the stability of supersonic flows is very intricate, there are (at least) three rough rules whose general validity has been confirmed in many independent studies: First, the instability of a shear flow becomes more benign with increasing Mach number: second, the most unstable modes are oblique; and, finally, there are several modes of instability--some of these may appear or disappear for certain ranges of Mach number and temperature. Because of this intricate behavior (which can be calculated numerically), it is desirable to obtain some theoretical understanding of certain limiting cases in order to provide a framework for a physical understanding.

The present study deals with the (nonlinear) stability of a supersonic mixing layer in the long-wave limit. The classic work in this general area is due to Miles (1958) and Fejer \& Miles (1963), who examined the small-disturbance (i.e. linearized) motion of a plane vortex sheet that is subjected to two- and three-dimensional disturbances. respectively. They found that the vortex sheet becomes marginally stable above a critical supersonic Mach number, and this Mach number is inversely proportional to $\cos \theta$, where $\theta$ is the propagation angle of the oblique mode. In addition, there are three of these marginally stable modes, which we label according to their phase speeds as the fast, slow, and intermediate modes. These classic results of Miles are clearly consistent with the previously stated rough guides for supersonic instabilities.

On the other hand, the numerical studies of Jackson \& Grosch (1989) indicate that the slow and fast modes are actually unstable in a mixing layer of finite (but small) thickness, with the growth rates depending on the square of the small wavenumber in the long-wave limit. while the frequency is directly proportional to the wavenumber itself. Therefore, the time scale associated with the exponential growth of the 
disturbance is very much longer than its period of oscillation. Thus, asymptotic analysis (e.g. multiple scales) can be used to describe the instability of the base flow. Furthermore. the effects of small nonlinearities may be incorporated into our analysis in order to affect the growth of the fundamental disturbance through cubic interactions.

The present study is very different from that of Artola \& Majda (1987). They examined the resonant response of a supersonic vortex sheet to an incident acoustic wave and determined the conditions for the evolution of a kink mode (i.e. a discontinuity in the slope of the vortex sheet). The principal difference is that Artola \& Majda were not concerned with the details of continuous base velocity and temperature profiles; their assumed wavelengths are so long that. for all practical purposes, the mixing layer appears to have negligible thickness. That is, they were interested in the nonlinear evolution of the vortex sheet as if it were a sharp contact discontinuity separating the two uniform external streams. This, of course, is not the case for the problem here, in which the wavenumbers (or frequencies) are assumed to be somewhat higher so that the nonlinear evolution of the disturbance is entirely dictated by the details of the continuous base flow profiles, especially in the vicinity of the critical level. At this level, the convection speed of the instability mode matches the local fluid speed.

It should be clear from the preceding remarks that the importance of the nonlinearities in the present study has its roots in the critical layer where the instability mode is approximately steady (in a reference frame moving at the convection speed). Thus, the familiar nonlinear terms in the usual substantial derivative cannot be ignored in this layer with respect to the unsteadiness; in essence, we have a nonlinear critical layer.

The importance of critical layers in the stability of shear flows has been recognized for a long time (Maslowe 1981). Usually critical layers arise for instability modes near the neutral wavenumber (or frequency), but they can also arise at low frequencies in 
incompressible or compressible wall-bounded flows (Goldstein, Durbin \& Leib 1987; Gajjar 1993). However, they cannot occur in an incompressible mixing layer, where the instability is of the Kelvin-Helmhoitz type, because both the growth rate and frequency are proportional to the (small) wavenumber.

The asymptotic form of the nonlinearity in the critical layer may manifest itself in several canonical forms, depending on the precise balance between the (slight) unsteadiness, the nonlinear terms, and (possibly) the viscous terms in the equations of motion. One of these canonical forms is the classical Landau-Stuart cubic term in the evolution equation for the amplitude of the fundamental (Drazin \& Reid, 1981, p. 370). Other forms, probably much less familiar but equally important and ubiquitous, are the strongly nonlinear phase-jump condition discovered by Benney \& Bergeron (1969) and subsequently developed by Stewartson (1981), Goldstein et al. (1987), and others, and the Hickernell (1984) cubic integral term in the amplitude equation.

In order to describe the evolution of a symmetric pair of interacting oblique instability modes on a shear flow, the integro-differential equation of Hickernell, or its variants, appears to be the most prevalent (Goldstein \& Choi 1989; Goldstein \& Leib 1989; Goldstein \& Lee 1992). In this case, the nonlinearity in the critical layer occurs in a hierarchical fashion: The lowest-order interaction of the fundamental mode with itself generates the mean flow distortion and the first harmonic, and a subsequent interaction of these newly generated disturbances with the fundamental reproduces the latter at cubic order in the (small) amplitude. For a properly posed physical problem involving spatial instability, each member of this hierarchy of equations is solved with vanishing boundary conditions imposed far upstream. The respective solutions are expressible in terms of integrals of the yet-unknown amplitude, and the products of these integrals, caused by the nonlinearities, ultimately yield the integro-differential equation for the amplitude. 
Since the flow outside the critical layer is unsteady, the nonlinear terms are relatively unimportant here, so the flow is governed by linearized equations. Because of the very tedious and distracting algebraic complexity that arises in the nonlinear problem, we first obtain the linearized solutions. These solutions will be valid everywhere (including the critical layer at lowest order). Then, in $\$ 7$, we calculate the nonlinear terms in the critical layer and determine the effects of these terms on the amplitude equation. The key result is (44).

The outline of the paper is as follows: The problem is formulated in 52 , and the scalings associated with the asymptotic analysis are introduced. It is shown that the cross-stream behavior of a low-frequency instability mode is determined by a triplelayer structure in the sense of matched asymptotic expansions. The relevant solutions in each of these layers are derived in $\$ \$ 3,4$, and 5 ; this discussion culminates in amplitude equation ( $31 a$ ) for the linearized problem.

In 86 , the amplitude scaling for the nonlinear problem is introduced. This is done by requiring that the slight unsteadiness of the fundamental (i.e. its weak instability) be balanced by the nonlinear convective derivative. as well as other nonlinearities in the equations of motion. The resultant nonlinear equations in the critical layer are solved in $\$ 7$ and in Appendix B. The net result is the appearance of one extra term in $(31 a)$; the normalized form of this term is given by the integral in (44). This is the final theoretical result--it includes cubic-type integrals of the amplitude over the entire life of the instability mode.

In closing this section, there are three points that we wish to emphasize. First, the backbone of the nonlinear theory presented herein is the linear theory. The latter provides a linear differential equation for the amplitude. Once the nonlinearities in the critical layer are incorporated into the analysis via the distinguished scaling of the (small) characteristic amplitude with respect to the (small) characteristic frequency, the amplitude equation is changed into a nonlinear integro-differential equation. 
Second, the lowest-order solution in the critical layer may be written as the corresponding solution in Goldstein \& Lee (1992), denoted by GL, plus an extra term that is proportional to a parameter $\Lambda$. In other words, if we (artificially) set $\Lambda=0$, we recover the results of Goldstein \& Lee for the flow field of the oblique modes. Subsequent products of our solutions always reproduce GL plus some extra terms that are proportional to suitable powers of $\Lambda$. In order to simplify the presentation of the nonlinear results, we only give these extra terms in Appendix B. Implicit is the understanding that the GL terms are available in the literature; in fact, both authors of this paper derived all terms independently. Agreement was obtained on all fronts, including with GL (Lee, private communication).

Finally, we establish that a nonlinear integro-differential equation describes the evolution of the amplitude at low frequencies. It is well known that a similar equation also holds in the vicinity of the neutral point. Since the growth rates are (numerically) small at large Mach numbers, it appears plausible that this type of nonlinear equation should hold for the entire frequency range. Thus we speculate that the qualitative behavior of a symmetric pair of oblique modes may be understood from an asymptotic analysis of this type.

\section{Formulation of problem}

Consider a unidirectional, inviscid, and parallel shear flow with velocity $U(y)$ i. pressure equal to unity, and density and temperature denoted by $\rho_{0}(y)$ and $T_{0}(y)$. respectively. Arbitrary perturbations in this base flow satisfy

$$
\begin{array}{lc}
\text { continuity: } & \frac{\mathrm{D} \rho}{\mathrm{D} t}+\mathbf{u} \cdot \nabla \rho+\rho_{0} \nabla \cdot \mathbf{u}+\sim \rho_{0}^{\prime}+\rho \nabla \cdot \mathbf{u}=0, \\
\text { momentum: } & \frac{\mathrm{Du}}{\mathrm{D} t}+\mathbf{u} \cdot \nabla \mathbf{u}+\sigma U^{\prime} \mathbf{i}=-\frac{1}{\gamma M^{2}} \frac{\nabla p}{\rho_{0}+\rho}, \\
\text { energy: } & \frac{\mathrm{D} p}{\mathrm{D} t}+\mathbf{u} \cdot \nabla p+\gamma(1+p) \nabla \cdot \mathbf{u}=0 . \\
\text { state: } & p=\rho T_{0}+\rho_{0} T+\rho T .
\end{array}
$$


where $\mathbf{u}=(u, v, w), p, \rho$, and $T$ are the perturbations in velocity, pressure, density, and temperature, and the prime denotes differentiation of a function with respect to its argument (here, it is $y$ ). The effects of dissipation and gravity are ignored, and we use

$$
\frac{\mathrm{D}}{\mathrm{D} t}=\frac{\partial}{\partial t}+U \frac{\partial}{\partial x}
$$

to denote the time derivative following the base flow. The geometry is defined in figure $1 ; \mathbf{x}=(x, y, z)$ is a Cartesian coordinate system, $t$ denotes time, and $\nabla=\mathrm{i}(\partial / \partial x)+$ $\mathbf{j}(\partial / \partial y)+\mathbf{k}(\partial / \partial z)$ is the gradient operator. The equation of state for the base flow implies $\rho_{0} T_{0}=1$.

All variables are considered nondimensional unless otherwise indicated. Lengths and velocities are normalized by a reference length and a reference speed $\left(L_{\text {ref }}, U_{\text {ref }}\right)$, respectively. These quantities measure a characteristic thickness and a characteristic speed of the mixing layer. For the purpose of developing the analysis, there is no need to be more specific about these quantities, whose ratio, $L_{\text {ref }} / U_{\text {ref }}$. provides a transit time for the normalization of time, $t$. Density, pressure, and temperature are normalized by their (dimensional) values in the upper stream at $y=+\infty$, but the undisturbed pressure is actually a constant (set to unity) throughout the mixing layer (as noted earlier). Thus, $\rho_{0}(+\infty)=T_{0}(+\infty)=1$, and $T_{0}(-\infty)=$ const. actually represents the temperature ratio across the mixing layer. We use the notation $(\cdot)_{ \pm}$in several different ways: when applied to the base flow it denotes the value of a quantity, $(\cdot)$, at $y= \pm \infty$; sometimes this value may be unity (e.g. $\rho_{0_{+}}$) but, in order to subsequently write our formulae economically and "symmetrically" with respect to the variables in the upper and lower streams, we do not evaluate $(\cdot)_{+}$explicitly.

In view of our nondimensionalization, $M=U_{\text {ref }} / a_{\text {ref }}$, where $a_{\text {ref }}=\sqrt{\gamma \mathscr{R} T_{\text {ref }}}$ is the (dimensional) unperturbed speed of sound in the upper stream. $T_{\text {ref }}$ is the corresponding temperature, and $\mathscr{R}$ is the (dimensional) gas constant. Clearly, $M$ is a characteristic Mach number for the flow. Our compressible mixing layer is assumed to be an ideal 
gas with constant specific heat capacities, whose usual ratio yields the isentropic exponent $\gamma=$ const. $=1.4$. Note that the energy equation $(1 c)$ is actually a combination of the first law of thermodynamics, the definition of entropy, and the equations of continuity and state. This combination is convenient to use because, in principle. $(1 a-c)$ provide $\rho, \mathbf{u}$, and $p$, and $(I d)$ yields $T$. Therefore, by using this formulation, we obtain a certain amount of decoupling among the equations.

To be specific, let the perturbations in the parallel shear flow be caused by a small-amplitude (of characteristic size $0<\epsilon \ll 1$ ), three-dimensional disturbance. Let this excitation occur far upstream (essentially at $x \rightarrow-\infty$ ) in the form of two linear instability modes of equal (complex) amplitude and of identical (real) frequency, but of opposite spanwise (i.e. z) wavenumbers. which we will assume to be real. Suppose further that the frequency of excitation is small. Without loss of generality, we take $\Delta U=U_{+}-U_{-}>0$ and, in order to ensure that the mixing layer is convectively unstable. $U_{-}$is considered sufficiently positive (Huerre \& Monkewitz 1990).

In order to continue with our formulation, we invoke a number of facts on the behavior of low-frequency or long-wave instability modes in a supersonic mixing layer at high Mach numbers. An a priori acceptance of these facts by the reader facilitates the introduction of the scales for the asymptotic theory, the multi-layer structure of the flow in the cross-stream (i.e. y) variable, and a discussion of the cumulative role of the nonlinearities. However, the validity of these facts should become clear as we develop our analysis; for the time being, we use the corresponding vortex-sheet results to motivate the discussion.

First, an important similarity parameter that determines the growth rate of an instability mode in a compressible mixing layer is the Mach number: $m=M \Delta U$ (Jackson \& Grosch 1989; Balsa \& Goldstein 1990). Second, when $m$ is larger than a critical value, say $m_{\mathrm{cr}}$, the mixing layer is marginally stable in the classical vortex-sheet limit (Miles 1958; Fejer \& Miles 1963). The actual value of $m_{\mathrm{cr}}$ depends on the 
temperature ratio across the mixing layer and the spanwise wavenumber of the disturbance. For example, for a two-dimensional mode and a temperature ratio of unity, $m_{\mathrm{cr}}=2 \sqrt{2}$. In our analysis, we focus attention on mixing layers with $m>m_{\mathrm{cr}}$; we call these mixing layers "highly" supersonic, although we are not examining the "hypersonic limit" $m \rightarrow \infty$. Third, these marginally stable vortex-sheet modes convect downstream at speed $U_{c}=O(1)$ (of course, $U_{-}<U_{c}<U_{+}$) such that $\left|U_{ \pm}-U_{\mathrm{c}}\right|$ is supersonic (in the usual aerodynamic meaning of this term) with respect to the upper/lower streams, respectively.

Consider now a low-frequency excitation of a highly supersonic mixing layer. To be precise, let $\delta=O(1)$ be the vorticity thickness of the layer and $\lambda$ be the characteristic wavelength of the instability mode: we shall examine the nonlinear spatial evolution of a pair of oblique modes when $\delta / \lambda=O(\sigma) \ll 1$, where $\sigma=\omega / U_{c}$. Here $\omega$ denotes the radian frequency of excitation, and we may think of $\sigma$ as the streamwise wavenumber. These oblique modes are actually unstable, with growth rates of $O\left(\sigma^{2}\right)$. Therefore, they appear marginally stable only in the classical vortex-sheet limit (i.e. as $\sigma \rightarrow 0$ in some sense; see \$3).

These considerations imply that the complex phase speed, $c$, of a low-frequency instability mode is of the form

$$
c=U_{\mathrm{c}}+\sigma(\cdot)+\ldots
$$

where (.) stands for some complex number. Therefore, the thickness of the critical layer is $O(\sigma)$. This layer is centered on the critical level $y=y_{\mathrm{c}}$, where $U\left(y_{\mathrm{c}}\right)=U_{\mathrm{c}}$. We emphasize that $U_{c}$ is given by classical vortex-sheet theory (see (7a)) so that the last equality defines $y_{\mathrm{c}}$. Since, in a mixing layer, $U(y)$ is a monotonic function of $y$, each mode has one critical layer. We note that even if this were not the case, it would be possible to extend our analysis at the expense of algebraic complexity (see the recent work of Wu. Lee \& Cowley 1993 on the Stokes layer). Recall that a supersonic mixing layer may support two instability modes (the slow and fast modes; see Jackson \& 
Grosch 1989); since these modes have separate critical layers, the nonlinear interaction between these modes is too weak to be considered in our analysis. We shall justify this remark $a$ posteriori in $\S 6$. Thus, the nonlinear interactions that we study arise from modes that share a single critical layer.

In order to describe the structure of the perturbations in the cross-stream coordinate. $y$. the method of matched asymptotic expansions is used. Because of the presence of three length scales $(\lambda \gg \delta \gg \sigma)$, the various layers in the flow are defined as follows:

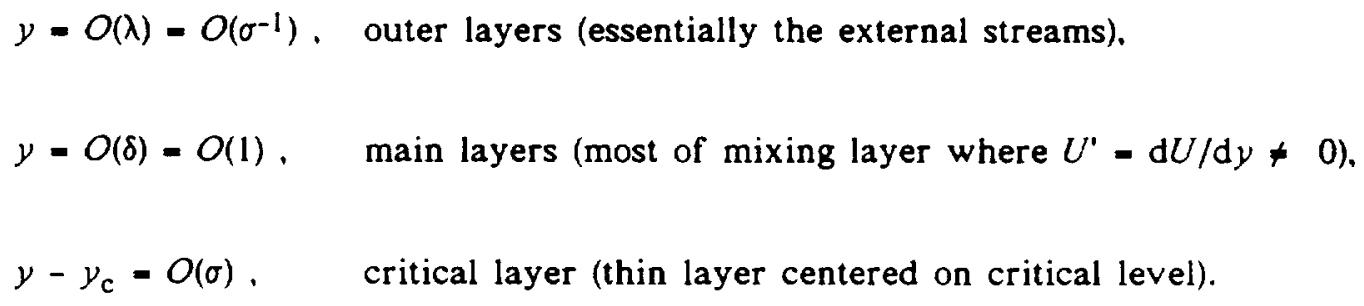

Thus, we have a triple-layer structure for the disturbance; the geometry of these layers is shown in figure 2.

The streamwise evolution of the unstable perturbations and the cumulative effect of the nonlinearities are described by the method of multiple scales. In view of (2). introduce

$$
\xi=\sigma\left(x-U_{c} t\right), \quad x_{1}=\sigma^{2} x
$$

for the "fast" and "slow" streamwise variables. Note that on the length scale of the mixing layer (i.e. its thickness), even $\xi$ is a "slow" variable; this is because we have long waves, which produce a gently varying disturbance in the $x$-direction. The streamwise oscillations of the instability wave occur on the space variable $\xi$, and the slow exponential growth and the cumulative nonlinear interaction between the oblique modes occur on the (even) slower variable $x_{1}$. In keeping with the ideas of multiple scales, we note 


$$
\frac{\partial}{\partial x} \rightarrow \sigma \frac{\partial}{\partial \xi}+\sigma^{2} \frac{\partial}{\partial x_{1}}
$$

and, for spatial instability,

$$
\frac{\partial}{\partial t}=-\sigma U_{\mathrm{c}} \frac{\partial}{\partial \xi}, \quad \frac{\partial}{\partial z}=\sigma l \frac{\partial}{\partial \zeta}, \quad \zeta=\sigma l z
$$

where $0<\sigma l \ll 1$ is the spanwise wavenumber. The propagation angles of the two oblique modes are $\pm \tan ^{-1}(\sigma l / \sigma)=O(1)$. Note that $\partial / \partial \xi, \partial / \partial x_{1}$, and $\partial / \partial \zeta$ are scaled operators that produce an $O(1)$ effect when applied to the low-frequency perturbations in the mixing layer. These considerations are valid in all layers; the correct scaling of the cross-stream variable, $y$, depends on the specific layer under discussion (see figure 2).

\section{Outer layers}

The relevant length scale in the outer layers is the wavelength. This implies that the scaled cross-stream variable is $Y=O(y / \lambda)=\sigma y$. The outer layers are defined by the condition $Y=O(1)$; in these layers, $U=U(y)=U(Y / \sigma)=U_{ \pm}=$const. as $\sigma \rightarrow 0$, under the assumption that $U(y)$ approaches $U_{ \pm}$exponentially quickly as $y \rightarrow \pm \infty$. Similar remarks hold for the unperturbed density and temperature profiles. Thus, the outer layers are the two external streams in which the base flow is completely uniform; this is essentially the vortex sheet limit. The space derivatives $\partial / \partial x, \partial / \partial y$, and $\partial / \partial z$ are equally important.

All perturbation quantities (velocity, pressure, etc.) expand as

$$
\begin{aligned}
& \text { velocity: } \quad \mathbf{u}=(u, v, w)=\epsilon \sigma\left(\mathbf{u}^{(0)}+\sigma \mathbf{u}^{(1)}+\ldots\right) \text {. } \\
& \text { pressure: } \quad p=\epsilon \sigma\left(p^{(0)}+\sigma p^{(1)}+\ldots\right) \text {, etc. }
\end{aligned}
$$

where the superscripted quantities are $O(1)$. Since we are (at the moment) dealing with linearized theory (see the discussion at the end of the "Introduction"), the factor $\epsilon \sigma$ on 
the right-hand sides of (4) has no significance; it is introduced here for convenience in anticipation of the nonlinear problem, for which we need a characteristic amplitude $\epsilon \ll 1$. This is determined in $\$ 6$.

Using (3c, d), we may express our substantial derivative $(1 e)$ as

$$
\frac{\mathrm{D}}{\mathrm{D} t}=\frac{\partial}{\partial t}+U \frac{\partial}{\partial x}=\sigma\left\{\left(U_{ \pm}-U_{\mathrm{c}}\right) \frac{\partial}{\partial \xi}+\sigma U_{ \pm} \frac{\partial}{\partial x_{1}}\right\}
$$

After substituting $(4,5)$ into $(1 a-d)$ and collecting terms with like powers of $\sigma$, we find that the lowest-order perturbations are given by a slight generalization of the Ackeret solution of classical supersonic aerodynamics. Thus,

$$
\begin{aligned}
p^{(0)}-\gamma M^{2} \mathcal{L}\left(x_{1}\right) \exp \left[i\left(\xi-q_{ \pm} Y+\zeta\right)\right] \\
u^{(0)}=-\frac{T_{0_{ \pm}}}{U_{ \pm}-U_{\mathrm{c}}} \frac{p^{(0)}}{\gamma M^{2}} . \\
a^{(0)}=\frac{T_{0_{ \pm}} q_{ \pm}}{U_{ \pm}-U_{\mathrm{c}}} \frac{p^{(0)}}{\gamma M^{2}} \\
w^{(0)}=-\frac{\ell T_{0_{ \pm}}}{U_{ \pm}-U_{\mathrm{c}}} \frac{p^{(0)}}{\gamma M^{2}}
\end{aligned}
$$

where $i=\sqrt{-1}$,

$$
q_{ \pm}=\left(\beta_{ \pm}^{2}-\ell^{2}\right)^{1 / 2}>0
$$

and

$$
\beta_{ \pm}^{2}=\frac{M^{2}\left(U_{ \pm}-U_{c}\right)^{2}}{T_{0_{ \pm}}}-1
$$

We recognize $\beta_{ \pm}>0$ as the Prandtl-Glauert factor and recall that $q_{ \pm}$will be real above the critical Mach number (Fejer \& Miles 1963), so that the instability wave must satisfy a radiation condition at $Y= \pm \infty$. This has been incorporated into the solution represented by (6) in order to satisfy causality via "outgoing" waves. 
We may think of our lowest-order solution in the outer layers as a "marginally stable" vortex-sheet mode whose complex amplitude, $\mathcal{L}\left(x_{1}\right)$, actually changes very slightly (by $O(\sigma)$ ) over a streamwise distance of one wavelength. In order to get the solution in the upper (or lower) streams, we use the + sign (or - sign) throughout (6). Note that the amplitude $\mathcal{A}$ is identical in both streams (i.e. $\mathcal{A}_{+}-\mathcal{L}_{-}-\mathscr{A}$ ). This comes from the asymptotic matching of the solutions through the intervening layers, or simply from (6a) and the physical observation that the lowest-order perturbation pressure must be continuous "across the vortex sheet."

In order to make a closer connection with classical supersonic aerodynamics, we observe that, at lowest order, the outer field is produced by a "wavy wall" whose vertical displacement may be estimated. Using (4a), (5), and (6c), we find the displacement

$$
\epsilon \frac{T_{0_{ \pm}} q_{ \pm}}{i\left(U_{ \pm}-U_{c}\right)^{2}} \mathcal{L}\left(x_{1}\right) \exp [i(\xi+\zeta)]
$$

Clearly, this "wall" has a unique displacement; therefore.

$$
\frac{T_{0_{+}} q_{+}}{\left(U_{+}-U_{\mathrm{c}}\right)^{2}}=\frac{T_{0_{-}} q_{-}}{\left(U_{-}-U_{\mathrm{c}}\right)^{2}} .
$$

Equation (7a) serves as the dispersion relation whose solutions yield the convection speed, $U_{c}$, of the instability modes as a function of the spanwise wavenumber and the properties of the external streams. Although it is possible to obtain (7a) from the asymptotic matching of the solutions across the various layers, it is helpful to have this result at hand in order to simplify the subsequent algebra. In this regard, define

$$
\Lambda_{ \pm}=\Lambda=\frac{T_{0_{ \pm}} q_{ \pm}}{\left(U_{ \pm}-U_{c}\right)^{2}}>0
$$

We call $\Lambda$ the vortex-sheet displacement parameter; its effect is felt in the other layers as well and shows up in the critical layer in the modification of the solution. 
As expected, the solution at the next order is considerably more complicated: the homogeneous solution is essentially in the form of (6) with $\mathscr{B} \pm$ playing the role of $\mathcal{A}$. and the particular solution has a secularity in $Y$. This secularity is well known in lowfrequency theories and is completely unimportant for our purposes. In any case, it may be eliminated by further subdividing the outer layers into additional layers; the secularity is due to the conversion of a slowly decaying exponential in $Y$ (due to the fact that the wave speed, $c$, has a small imaginary part) to its linear approximation by its Taylor series.

The second-order perturbations account for the fact that the outer flow is slightly unsteady in a reference frame that translates with velocity $U_{c} i$. They are given by

$$
\begin{gathered}
p^{(1)}=\gamma M^{2}\left\{\mathscr{B}_{ \pm}\left(x_{1}\right)-\mathscr{K} \pm \mathcal{L}^{\prime}\left(x_{1}\right)\right\} \exp \left[i\left(\xi-q_{ \pm} Y+\zeta\right)\right], \\
u^{(1)}=-\frac{T_{0_{ \pm}}}{U_{ \pm}-U_{\mathrm{c}}}\left\{\frac{p^{(1)}}{\gamma M^{2}}+\frac{i U_{c}}{U_{ \pm}-U_{\mathrm{c}}} \mathcal{M}^{\prime}\left(x_{1}\right) \exp \left[i\left(\xi-q_{ \pm} Y+\zeta\right)\right]\right\}, \\
u^{(1)}=\frac{T_{0_{ \pm}} q_{ \pm}}{U_{ \pm}-U_{c}}\left\{\frac{p^{(1)}}{\gamma M^{2}}-\frac{i\left(U_{\mathrm{c}}+\ell^{2} U_{ \pm}\right)}{q_{ \pm}^{2}\left(U_{ \pm}-U_{\mathrm{c}}\right)} \mathcal{K}^{\prime}\left(x_{1}\right) \exp \left[i\left(\xi-q_{ \pm} Y+\zeta\right)\right]\right\}, \\
w^{(1)}=-\frac{\ell T_{0_{ \pm}}}{U_{ \pm}-U_{\mathrm{c}}}\left\{\frac{p^{(1)}}{\gamma M^{2}}+\frac{i U_{ \pm}}{U_{ \pm}-U_{\mathrm{c}}}-\mathcal{L}^{\prime}\left(x_{1}\right) \exp \left[i\left(\xi-q_{ \pm} Y+\zeta\right)\right]\right\} .
\end{gathered}
$$

where

$$
\mathscr{K}_{ \pm}=\frac{\beta_{ \pm}^{2}}{q_{ \pm}}+\frac{M^{2} U_{c}\left(U_{ \pm}-U_{c}\right)}{q_{ \pm} T_{0_{ \pm}}}
$$

and $\mathcal{C}^{\prime}=\mathrm{d} \mathcal{C} / \mathrm{d} x_{1}$. At this stage, $\mathscr{B}_{ \pm}\left(x_{1}\right)$ are two arbitrary functions, which are needed to carry out the asymptotic matching.

From the observation that the flow is homentropic in each of the outer layers (albeit with different entropies), the perturbation density and temperature at the two leading orders are 


$$
\begin{gathered}
\rho^{(j)}-\frac{p^{(j)}}{\gamma T_{0_{ \pm}}}, \\
T^{(j)}=\frac{\gamma-1}{\gamma} T_{0_{ \pm}} p^{(j)} .
\end{gathered}
$$

for $j=0.1$.

This concludes our discussion of the flow in the outer layers. This flow is extremely simple: it is essentially a small-disturbance and slightly unsteady flow. As we shall see, the introduction of nonlinearities requires no modifications in the solutions at the orders considered. In other words. the disturbances in the outer layers are governed by linear dynamics to the required order of accuracy. Thus, superposition remains valid and, because of this, we have focused our attention on only one oblique mode, whose propagation vector points into the first quadrant of $(x, z)$ space. Furthermore, we have written the solutions in complex form, with the usual convention in mind that the physical solution is given by the real (or imaginary) part of these complex expressions. For the oblique mode whose propagation vector lies in the second quadrant, replace $\ell$ by $(-\ell)$ and $\zeta$ by $(-\zeta)$. We now turn our attention to the main layers, in which the gradients of the base flow profiles begin to play a role, although nonlinear effects are still unimportant.

\section{Main shear layers}

The relevant length scale in the two main layers is the characteristic thickness of the mixing layer. This implies that the scaled cross-stream variable is $y / \delta$, or simply $y$, since $\delta=O(1)$. The main layers are defined by the condition $y=O(1)$, although the critical layer, sandwiched between them. is excluded. We must account for the dependence of $U, \rho_{0}$, and $T_{0}$ on $y$ in these layers.

The perturbation quantities expand differently than in the outer layers. This signifies completely different physics. As we have seen from the outer solutions, 
disturbances of $O(\epsilon \sigma)$ produce a vortex-sheet displacement of $O(\epsilon \sigma / \sigma)=O(\epsilon) \gg>(\epsilon \sigma)$. Such a displacement of a material volume in a non-uniform base flow causes perturbations of $O(\epsilon)$. Therefore,

$$
u=\epsilon\left(\hat{u}^{(0)}+\sigma \hat{u}^{(1)}+\ldots\right)
$$

with similar expansions for the perturbation density and temperature. On the other hand. since $\delta / \lambda=O(\sigma) \ll 1$. the main layers are "thin" so that the pressure is impressed on them by the outer layers. Thus.

$$
p=\epsilon \sigma\left(\hat{p}^{(0)}+\sigma \hat{p}^{(1)}+\ldots\right)
$$

and

$$
\sigma=\epsilon \sigma\left(\hat{v}^{(0)}+\sigma \hat{v}^{(1)}+\ldots\right)
$$

since, in a thin layer, $w=O(u \delta / \lambda)$. Finally.

$$
w=\epsilon \sigma\left(\hat{w}^{(0)}+\sigma \hat{w}^{(1)}+\ldots\right)
$$

since spanwise velocity perturbations must originate in spanwise pressure gradients (the previously alluded to displacement effect is absent since the base velocity is along the $x$-axis). The caret indicates that a variable is written for the main layers, and the superscript designates the place of a term in the expansion. As usual, $(\cdot)^{(0)}$ and $(\cdot)^{(1)}$ are $O(1)$. The ellipses in (10) stand for higher-order terms that are not needed in this study. Using ( $3 c, d)$, we express our substantial derivative, (le), as

$$
\frac{\mathrm{D}}{\mathrm{D} t}=\frac{\partial}{\partial t}+U \frac{\partial}{\partial x}=\sigma\left\{\left(U-U_{\mathrm{c}}\right) \frac{\partial}{\partial \xi}+\sigma U \frac{\partial}{\partial x_{1}}\right\} .
$$

and, after substituting (10) and (11) into the linearized form of (1), we may obtain a set of equations for $\hat{(\cdot)}^{(0)}$ and $\hat{(\cdot)}^{(1)}$.

The lowest-order equations are simple to solve. For example, $\partial \hat{p}^{(0)} / \partial y=0$ so that $\hat{p}^{(0)}$ is a function of $\xi . \zeta$, and $x_{1}$. This function is determined from matching with the 
pressure in the outer layers. Thus,

$$
\hat{p}^{(0)}=\gamma M^{2} \mathcal{L}\left(x_{1}\right) \exp [i(\xi+\zeta)]
$$

and, from the spanwise momentum equation, we get

$$
\hat{w}^{(0)}=-\frac{\ell T_{0}}{U-U_{\mathrm{c}}} \mathcal{L}\left(x_{1}\right) \exp [i(\xi+\zeta)]
$$

Note that $(12 b)$ matches with $(6 d)$; it also blows up as $y \rightarrow y_{\mathrm{c}}$. This establishes the need for the critical layer and a rescaling of the solutions there. This is discussed in 85 .

Similarly, by solving the remaining equations of motion, we obtain

$$
\begin{aligned}
& \hat{u}^{(0)}=-U^{\prime} G_{ \pm}^{(0)}\left(\xi, \zeta, x_{1}\right), \\
& \hat{T}^{(0)}=-T_{0}^{\prime} G_{ \pm}^{(0)}\left(\xi, \zeta, x_{1}\right), \\
& \hat{\rho}^{(0)}=-\rho_{0}^{\prime} G_{ \pm}^{(0)}\left(\xi, \zeta, x_{1}\right) .
\end{aligned}
$$

and

$$
\hat{\vartheta}^{(0)}=\left(U-U_{c}\right) G_{ \pm \xi}^{(0)}\left(\xi, \zeta, x_{1}\right)
$$

where $G_{ \pm}^{(0)}$ are two arbitrary functions of the indicated arguments (i.e. independent of y). The \pm signs refer to the values of these functions in the two main layers. one above and one below the critical layer, and the subscript $\xi$ on $G_{ \pm}^{(0)}$ denotes partial differentiation with respect to $\xi$. Also, $U^{\prime}=\mathrm{d} U / \mathrm{d} y, T_{0}^{\prime}=\mathrm{d} T_{0} / \mathrm{d} y$, and $\rho_{0}^{\prime}=\mathrm{d} \rho_{0} / \mathrm{d} y$.

The asymptotic matching of $(12 f)$ and $(6 c)$ immediately establishes

$$
G_{ \pm}^{(0)}=G^{(0)}=-i \Lambda \mathcal{A}\left(x_{1}\right) \exp [i(\xi+\zeta)]
$$

where $\Lambda$ is defined by $(7 b)$. Note that $(12 c, d, e)$ are perfectly consistent with our opening remarks of this section; Eulerian perturbations in the u-velocity, temperature, and density are proportional to the corresponding base flow gradients and arise from the displacement of a material volume in the nonuniform base flow. 
We are also interested in the solutions at the next order; it is precisely here that the main-layer expansion further breaks down as $y \rightarrow y_{c}$. The $y$-momentum equation may be solved to yield the pressure at second order

$$
\hat{p}^{(1)}=-\gamma M^{2} \frac{q_{ \pm} G_{\xi \xi}^{(0)}}{\Lambda}\left\{y+\int_{ \pm \infty}^{y}\left[\Gamma_{ \pm}(y)-1\right] \mathrm{d} y\right\}+\hat{p}_{ \pm}^{(1)}\left(\xi, \zeta, x_{1}\right)
$$

where

$$
\Gamma_{ \pm}(y)=\frac{\left[\frac{U(y)-U_{c}}{U_{ \pm}-U_{c}}\right]^{2}}{\frac{T_{0}(y)}{T_{0_{ \pm}}}}
$$

and $\hat{p}_{ \pm}^{(1)}$ are two arbitrary functions of their indicated arguments; they represent the homogeneous solution to the boundary layer equation $\partial / \partial y=0$. It is possible to determine them by matching the pressures in the main layers to those in the outer layers. We find

$$
\hat{p}_{ \pm}^{(1)}=\gamma M^{2} \mathscr{B}_{ \pm}\left(x_{1}\right) \exp [i(\xi+\zeta)]
$$

where $B_{ \pm}$was introduced in $(8 a)$ as part of the homogeneous solution in the outer layers at second order.

The spanwise gradients of the pressure given by $(14 a)$ drive the spanwise velocity component. The corresponding equation of motion may be solved for

$$
\hat{w}^{(1)}=-\frac{i \ell U T_{0}}{\left(U-U_{\mathrm{c}}\right)^{2}}-\mathcal{\ell}^{\prime}\left(x_{1}\right) \exp [i(\xi+\zeta)]-\frac{\ell T_{0}}{U-U_{\mathrm{c}}} \frac{\hat{p}^{(1)}}{\gamma M^{2}}
$$

Note that the first term on the right-hand side of (15) arises from the variation of $\hat{w}^{(0)}$ on the slow scale $x_{1}$, whereas the second term is due to spanwise pressure gradients. The spanwise velocity components in the outer and main layers automatically match. 
At second order, the streamwise and cross-stream velocity components are most easily obtained from the energy $(I c)$ and $x$-momentum $(1 b)$ equations. The algebra is quite involved; therefore, we provide an intermediate step that documents the coupled equations for $\hat{u}^{(1)}$ and $\hat{\sigma}^{(1)}$ :

$$
\begin{aligned}
& \frac{\partial \hat{u}^{(1)}}{\partial \xi}+\frac{\partial \hat{\sigma}^{(1)}}{\partial y}=-\frac{\partial \hat{u}^{(0)}}{\partial x_{1}}-\frac{U-U_{c}}{\gamma} \frac{\partial \hat{p}^{(0)}}{\partial \xi}-\ell \frac{\partial \hat{w}^{(0)}}{\partial \xi} \\
& \left(U-U_{c}\right) \frac{\partial \hat{u}^{(1)}}{\partial \xi}+U^{\prime} \hat{\sigma}^{(1)}=-U \frac{\partial \hat{u}^{(0)}}{\partial x_{1}}-\frac{T_{0}}{\gamma M^{2}} \frac{\partial \hat{p}^{(0)}}{\partial \xi} .
\end{aligned}
$$

After eliminating $\partial \hat{\sim}^{(1)} / \partial \xi$ from (16b) via $(16 a)$, substituting the lowest-order solutions (12) into the forcing terms, and solving. we obtain

$$
\begin{aligned}
\hat{\sigma}^{(1)}=\frac{i T_{0_{ \pm}}}{\left(U_{ \pm}-U_{c}\right)^{2}}\left\{\left(U-U_{c}\right) F_{ \pm}\left(\xi, y, \zeta, x_{1}\right)-U q_{ \pm} \mathcal{L}\left(x_{1}\right) \exp [i(\xi+\zeta)]\right\} \\
+\left(U-U_{\mathrm{c}}\right) G_{ \pm \xi}^{(1)}\left(\xi, \zeta, x_{1}\right),
\end{aligned}
$$

where

$$
\begin{gathered}
F_{ \pm}\left(\xi, y, \zeta, x_{1}\right)=\left\{\left(1+l^{2}\right) \int_{ \pm \infty}^{y}\left[\frac{1}{\Gamma_{ \pm}(y)}-1\right] \mathrm{d} y-y q_{ \pm}^{2}\right\} \mathcal{A}\left(x_{1}\right) \exp [i(\xi+\zeta)] \\
+q_{ \pm} \mathcal{C}^{\prime}\left(x_{1}\right) \exp [i(\xi+\zeta)] .
\end{gathered}
$$

and $G_{ \pm}^{(1)}$ is an arbitrary function of its arguments; the last term in $(17 a)$ represents the homogeneous solution.

The streamwise velocity component follows from $(16 a)$ or $(16 b)$ after eliminating $\hat{\sigma}(1)$ via $(17 a)$. We find

$$
\begin{gathered}
\hat{u}^{(1)}=-\frac{U^{\prime} T_{0_{ \pm}}}{\left(U_{ \pm}-U_{c}\right)^{2}} F_{ \pm}\left(\xi, y, \zeta, x_{1}\right)-\frac{T_{0}}{U-U_{c}} \mathcal{L}\left(x_{1}\right) \exp [i(\xi+\zeta)] \\
-U^{\prime} G_{ \pm}^{(1)}\left(\xi, \zeta, x_{1}\right) .
\end{gathered}
$$


The asymptotic matching of the cross-stream velocity component to that in the outer layers yields

$$
\begin{aligned}
G_{ \pm}^{(1)}= & -i \Lambda \mathscr{B}_{ \pm}\left(x_{1}\right) \exp [i(\xi+\zeta)] \\
& +\frac{\Lambda}{U_{ \pm}-U_{c}}\left[U_{c}-\frac{U_{c}+\ell^{2} U_{ \pm}}{q_{ \pm}^{2}}\right) \mathcal{L}^{\prime}\left(x_{1}\right) \exp [i(\xi+\zeta)] .
\end{aligned}
$$

As mentioned before. the solution in the main layer breaks down in the vicinity of the critical level where $\left(U-U_{c}\right)$ is small; see for example $(12 b)$. In order to repair the resultant disorder in the expansion, the critical layer is introduced. The behavior of the main solution at the edge of the critical layer is discussed more fully in Appendix A; such a discussion is essential in order to match the solutions in the critical and main layers.

\section{The critical layer}

The critical layer is centered on the critical level, $y=y_{c}$, and its thickness is of $O(\sigma)$. Therefore, introduce the critical layer coordinate

$$
\eta=\frac{y-y_{\varepsilon}}{\sigma}
$$

with $\eta=O(1)$, and expand the base flow in its Taylor series about the point $y=y_{c}$. We find, using (le),

$$
\frac{\mathrm{D}}{\mathrm{D} t}=\frac{\partial}{\partial t}+U \frac{\partial}{\partial x}=\sigma^{2}\left\{\mathscr{L}^{(0)}+\sigma \mathscr{L}^{(1)}+O\left(\sigma^{2}\right)\right\}
$$

where $\mathscr{L}^{(0)}$ and $\mathscr{L}^{(1)}$ are two linear operators defined by

$$
\begin{aligned}
& \mathscr{L}^{(0)}=U_{\mathrm{c}} \frac{\partial}{\partial x_{1}}+U_{\mathrm{c}}^{\prime} \eta \frac{\partial}{\partial \xi}, \\
& \mathscr{L}^{(1)}=\eta\left[U_{\mathrm{c}}^{\prime} \frac{\partial}{\partial x_{1}}+\frac{U_{\mathrm{c}}^{\prime \prime}}{2} \eta \frac{\partial}{\partial \xi}\right] .
\end{aligned}
$$

The notation $(\cdot)_{c}$ means that $(\cdot)$ is evaluated at $y=y_{c}$. 
Based on the discussion presented in $\$ 4$, we find that the perturbation quantities expand as

$$
\begin{aligned}
& u=\epsilon\left(\hat{u}^{(0)}+\sigma \hat{u}^{(1)}+\ldots\right) . \\
& w=\epsilon\left(\hat{w}^{(0)}+\sigma \hat{w}^{(1)}+\ldots\right) .
\end{aligned}
$$

with similar expressions for the density and temperature. whereas

$$
p=\epsilon \sigma\left(\hat{p}^{(0)}+\sigma \hat{p}^{(1)}+\ldots\right)
$$

and

$$
v=\epsilon \sigma^{2}\left(\hat{v}^{(0)}+\sigma \hat{v}^{(1)}+\ldots\right)
$$

Note that $(20 c, d)$ are entirely consistent with the fact that the critical layer is a thin boundary layer (in a generalized sense of this term), and the form of (20b) expresses the fact that the spanwise velocity component in the critical layer is $O(1 / \sigma)$ larger than that in the main layers (see $12(b))$.

The double caret indicates that a dependent variable is written for the critical layer, and the superscript denotes the place of a term in the expansion. As usual, $\hat{(\cdot)^{(0)}}$ and $\hat{(\cdot)^{(1)}}$ are $O(1)$, and they depend on the critical layer coordinate $\eta$ such that $\partial / \partial \eta$ of these terms are also of $O(1)$.

We now use (18)-(20) in the equations of motion, (1), in order to derive a sequence of equations for the flow in the critical layer. The simplest of these comes from the $y$ momentum equation and yields

$$
\frac{\partial \hat{p}^{(0)}}{\partial \eta}=\frac{\partial \hat{p}^{(1)}}{\partial \eta}=0
$$

so that $\hat{p}^{(j)}=f(j)\left(\xi, \zeta, x_{1}\right)(j=0,1)$; the actual function form may be obtained from matching with the main layers. Thus, using (12a) and $(14 a, c)$, we find

$$
\hat{p}^{(0)}=\gamma M^{2} \mathcal{M}\left(x_{1}\right) \exp [i(\xi+\zeta)] .
$$




$$
\begin{aligned}
\hat{p}^{(1)}=-i \gamma M^{2} q_{ \pm} & \left\{y_{c}+\int_{ \pm \infty}^{y_{c}}\left[\Gamma_{ \pm}(y)-1\right] \mathrm{d} y\right\} \ell\left(x_{1}\right) \exp [i(\xi+\zeta)] \\
& +\gamma M^{2} \mathscr{B}_{ \pm}\left(x_{1}\right) \exp [i(\xi+\zeta)] .
\end{aligned}
$$

Since there must exist a unique $\hat{p}^{(1)}$ that is independent of the label ( \pm ), the solvability of $(22 b)$ implies

$$
\nexists_{+}\left(x_{1}\right)-i Q_{+} \mathcal{L}\left(x_{1}\right)=B_{-}\left(x_{1}\right)-i Q_{-} \mathcal{L}\left(x_{1}\right)
$$

where

$$
Q_{ \pm}=\text {const. }=q_{ \pm}\left\{y_{\mathrm{c}}+\int_{ \pm \infty}^{y_{\mathrm{c}}}\left[\Gamma_{ \pm}(y)-1\right] \mathrm{d} y\right\} \text {. }
$$

Now recall from (8) that $\bar{D}_{\ddagger}$ are associated with the homogeneous solutions in the outer layers at $O(\sigma)$. These solutions merely reproduce the lowest-order solutions; in order to assign a unique gauge to the amplitude $\mathcal{L}\left(x_{1}\right)$, we set $B_{-}=0$. Thus, we interpret $\mathcal{t}$ as the amplitude of the instability mode (to the required order of accuracy) in the slow external stream, and $(23 a)$ defines $\mathscr{B}_{+}$.

The lowest-order spanwise velocity component is essentially generated by the $\zeta$-derivative of $\hat{p}^{(0)}$; we solve the leading-order $z$-momentum equation to obtain

$$
\hat{w}^{(0)}=-\frac{i \ell T_{0_{\mathrm{c}}}}{U_{\mathrm{c}}} W_{0}\left(\eta, x_{1}\right) \exp [i(\xi+\zeta)]
$$

where

$$
\begin{aligned}
& W_{\mathrm{n}}\left(\eta, x_{1}\right)=I_{\mathrm{n}}\left(\eta, x_{1}\right) \exp \left(-i \alpha \eta x_{1}\right), \\
& I_{\mathrm{n}}\left(\eta, x_{1}\right)=\int_{-\infty}^{x_{1}}\left(\hat{x}_{1}-x_{1}\right)^{\mathrm{n}} \mathcal{M}\left(\hat{x}_{1}\right) \exp \left(i \alpha \eta \hat{x}_{1}\right) \mathrm{d} \hat{x}_{1}, \quad n=0,1,2, \ldots,
\end{aligned}
$$

and $\alpha=U^{\prime}{ }_{\mathrm{c}} / U_{\mathrm{c}}$. This apparently cumbersome notation involving $W_{\mathrm{n}}$ and $I_{\mathrm{n}}$ turns out to be extremely useful for representing the hierarchy of solutions of the linear and 
nonlinear problems. Therefore, we introduce it at this stage of the analysis. Note that the asymptotic behavior of $W_{n}(n=0,1, \ldots)$ for large $|\eta|$ may be obtained by successive integrations by parts. For example,

$$
W_{0}\left(\eta, x_{1}\right)=\frac{\mathcal{L}\left(x_{1}\right)}{i \alpha \eta}-\frac{\mathcal{L}^{\prime}\left(x_{1}\right)}{(i \alpha \eta)^{2}}+O\left(\eta^{-3}\right)
$$

under the (correct) assumption that $\mathcal{L}\left(x_{1}\right)$ vanishes exponentially as $x_{1} \rightarrow-\infty$. Similar expressions may be obtained for $W_{\mathrm{n}}(n \geq 1)$ whose leading term is $O\left(1 / \eta^{1+n}\right)$.

The $x$-momentum and energy equations for $\hat{u}^{(0)}$ and $\hat{v}^{(0)}$ are coupled. However. an advantageous decoupling is effected if we first solve for $\partial \hat{u}^{(0)} / \partial \eta$. The relevant equation (essentially for $z$-vorticity) is

$$
\left[\frac{\partial}{\partial x_{1}}+\alpha \eta \frac{\partial}{\partial \xi}\right] \frac{\partial \hat{u}^{(0)}}{\partial \eta}=\alpha \ell \frac{\partial \hat{w}^{(0)}}{\partial \zeta}
$$

which expresses the fact that a $(z-z)$ normal strain stretches a spanwise vortex line and thereby changes the corresponding vorticity. The solution of (26a) may be integrated immediately with respect to $\eta$ to yield

$$
\hat{u}^{(0)}=\frac{i \ell^{2} T_{0_{c}}}{U_{c}} W_{0}\left(\eta, x_{1}\right) \exp [i(\xi+\zeta)]+i \Lambda U_{c}^{\prime} \mathcal{L}\left(x_{1}\right) \exp [i(\xi+\xi)]
$$

where the last term in (26b), the "constant" of integration, arises from matching (26b) and $(12 c)$. Finally, having determined $\hat{p}^{(0)}$ and $\hat{u}^{(0)}$, we obtain $\hat{v}^{(0)}$ from the lowest-order $x$-momentum equation

$$
\begin{gathered}
\hat{\vartheta}^{(0)}=-\frac{T_{0_{c}}\left(1+\ell^{2}\right)}{U_{c}^{\prime}} \mathcal{A}\left(x_{1}\right) \exp [i(\xi+\zeta)]-i \Lambda U_{c}\left[\mathcal{K}^{\prime}\left(x_{1}\right)\right. \\
\left.+i \alpha \eta \mathcal{M}\left(x_{1}\right)\right] \exp [i(\xi+\zeta)] .
\end{gathered}
$$

Note that $(26 c)$ automatically matches with $(12)$.

At this point in the analysis, $\mathcal{L}\left(x_{1}\right)$ is still an arbitrary function of its argument; in order to determine the dependence of the amplitude on the slow variable $x_{1}$, we must 
calculate the $O(\sigma)$ corrections to the critical layer solution. These are denoted by $(\hat{\sigma})(1)$ in (20); specifically, we need $\hat{u}^{(1)}$ in order to derive the amplitude equation.

The spanwise velocity component, $\hat{w}^{(1)}$, obeys the inhomogeneous equation

$$
\mathscr{L}^{(0)} \hat{w}^{(1)}=-\mathscr{L}^{(1)} \hat{w}^{(0)}-\frac{\ell}{\gamma M^{2}} \frac{\partial}{\partial \zeta}\left[T_{0_{c}} \hat{p}^{(1)}+T_{0_{c}}^{\prime} \eta \hat{p}^{(0)}\right]
$$

whose right-hand side is known from the lowest-order critical layer solutions. $(22 a, b)$ and $(24 a)$. In order to solve $(27 a)$, we observe the identity

$$
\left[\frac{\partial}{\partial x_{1}}+i \alpha \eta\right) W_{1}\left(\eta, x_{1}\right)=-W_{0}\left(\eta, x_{1}\right)
$$

and write the solution

$$
\begin{aligned}
\hat{w}^{(1)} \exp [-i(\xi+\zeta)]= & \frac{l T_{0_{c}} \eta^{2}}{U_{c}}\left(\frac{U_{c}^{\prime \prime}}{2 U_{c}}-\alpha^{2}\right) W_{1}\left(\eta, x_{1}\right)-\frac{l T_{0_{c}} Q_{-}}{U_{c}} W_{0}\left(\eta, x_{1}\right) \\
& +\frac{i l \eta}{U_{c}}\left(\alpha T_{0_{c}}-T_{0_{c}}^{\prime}\right) W_{0}\left(\eta, x_{1}\right) .
\end{aligned}
$$

It is possible to show that the two-term critical-layer solution for the spanwise velocity component matches with that in the main layers in the sense of the asymptotic matching principle of Van Dyke (i.e. 2-main of (2-critical) $=2$-critical of (2-main)). For this purpose, the behavior of the solutions in the main layers near the edges of the critical layer is derived in Appendix A.

As before, it is simpler to solve for $\partial \hat{u}^{(1)} / \partial \eta$, the $O(\sigma)$ correction to the spanwise vorticity, rather than directly for $\hat{\boldsymbol{u}}^{(1)}$. The governing equation is

$$
\mathscr{L}^{(0)} \frac{\partial \hat{u}^{(1)}}{\partial \eta}=\ell \frac{\partial}{\partial \xi}\left[U_{c}^{\prime} \hat{w}^{(1)}+U_{c}^{\prime \prime} \eta \hat{w}^{(0)}\right]-\mathscr{L}^{(1)} \frac{\partial \hat{u}^{(0)}}{\partial \eta}-U_{c}^{\prime \prime} \hat{\sigma}^{(0)}-\frac{T_{0_{c}}^{\prime}}{\gamma M^{2}} \frac{\partial \hat{p}^{(0)}}{\partial \xi}
$$

whose right-hand side contains the vortex stretching and displacement effects, as well as that of the baroclinic torque (the last term). 
If we substitute the previously determined solutions into the right-hand side of (28a), we obtain, after a modest amount of algebra,

$$
\begin{gathered}
\exp [-i(\xi+\zeta)] \mathscr{L}^{(0)} \frac{\partial \hat{u}^{(1)}}{\partial \eta}=i \ell^{2} \alpha T_{0_{c}} \eta^{2}\left(\frac{U_{c}^{\prime \prime}}{U_{c}}-2 \alpha^{2}\right) W_{1}\left(\eta, x_{1}\right)-\left[i \ell^{2} \alpha T_{0_{c}} Q_{-}+2 \ell^{2} \alpha^{2} T_{0_{c}} \eta\right. \\
\left.-\frac{\ell^{2} \eta}{U_{c}}\left(U_{c}^{\prime} T_{0_{c}}^{\prime}+U_{c}^{\prime \prime} T_{0_{c}}\right)\right] W_{0}\left(\eta, x_{1}\right)+i T_{0_{c}}\left\{\frac{U_{c}^{\prime \prime}\left(1+\ell^{2}\right)}{U_{c}^{\prime}}-\frac{T_{0_{c}}^{\prime}}{T_{0_{c}}}\right\} \mathcal{L}\left(x_{1}\right) \\
+i \Lambda U_{c}^{\prime \prime} U_{c} \exp \left(-i \alpha \eta x_{1}\right) \frac{\partial}{\partial x_{1}}\left[\mathcal{L}\left(x_{1}\right) \exp \left(i \alpha \eta x_{1}\right)\right] .
\end{gathered}
$$

whose solution is

$$
\begin{gathered}
\frac{\partial \hat{u}^{(1)}}{\partial \eta} \exp [-i(\xi+\zeta)]=-\frac{i \ell^{2} \alpha T_{0_{c}} U_{c}^{\prime \prime}}{2 U_{c}^{2}} \eta^{2} W_{2}\left(\eta, x_{1}\right)-\frac{\ell^{2}}{U_{c}^{2}}\left(U_{c}^{\prime} T_{0_{c}}^{\prime}+U_{c}^{\prime \prime} T_{0_{c}}\right) \eta W_{1}\left(\eta, x_{1}\right) \\
+\frac{i T_{0_{c}}}{U_{c}}\left\{\frac{U_{c}^{\prime \prime}\left(1+\ell^{2}\right)}{U_{c}^{\prime}}-\frac{T_{0_{c}}^{\prime}}{T_{0_{c}}}\right\} W_{0}\left(\eta, x_{1}\right)-\frac{\ell^{2} \alpha^{2} T_{0_{c}} \eta}{U_{c}} \frac{\partial W_{2}}{\partial x_{1}} \\
+\frac{i \ell^{2} \alpha T_{0_{c}} Q}{U_{c}} W_{1}\left(\eta, x_{1}\right)+i \Lambda U_{c}^{\prime \prime} \mathcal{M}\left(x_{1}\right) .
\end{gathered}
$$

In deriving (28c), we have made use of an identity, which is a slight generalization of (27b), involving the higher-order $W_{\mathrm{n}}$ 's.

In order to carry out the asymptotic matching with the main layers. we first reconstruct the streamwise velocity component

$$
\hat{u}^{(1)}(\eta)-\hat{u}^{(1)}(0)=\int_{0}^{\eta} \frac{\partial \hat{u}^{(1)}}{\partial \eta} \mathrm{d} \eta
$$

where, for emphasis and brevity of notation, we only show the $\eta$-argument of $\hat{u}^{(1)}$ (of course, the other arguments are $\xi, \xi$, and $\left.x_{1}\right)$. We cannot let $\eta \rightarrow \pm \infty$ in $(29 a)$ in order to determine the behavior of $\hat{u}^{(1)}$ at the edges of the critical layer for the purpose of matching, since the above integral is divergent at the upper limit. 
In order to get around this difficulty, introduce

$$
Z(\eta)=\frac{\partial \hat{u}^{(1)}}{\partial \eta}-\left\{i \Lambda U_{c}^{\prime \prime}-\frac{T_{0_{c}} B}{U_{c}^{\prime}}\left(1+\ell^{2}\right) \frac{\eta}{1+\eta^{2}}\right\} \mathcal{L}\left(x_{1}\right) \exp [i(\xi+\zeta)],
$$

where

$$
B=\left[\frac{T_{0}^{\prime}}{T_{0}}-\frac{U^{\prime \prime}}{U^{\prime}}\right]_{y-y_{c}}
$$

Observe that it is possible to show from $(28 c)$ and the asymptotic behavior of $W_{\mathrm{n}}$ that $Z(\eta)$ is analytic for all (real) $\eta$ and is integrable at $\pm \infty$. Therefore, we rewrite $(29 a)$ as

$$
\begin{aligned}
\hat{u}^{(1)}(\eta) & =\hat{u}^{(1)}(0)+\int_{0}^{ \pm \infty} Z(\eta) \mathrm{d} \eta+\int_{ \pm \infty}^{\eta} Z(\eta) \mathrm{d} \eta \\
& +\left\{i \Lambda U_{\mathrm{c}}^{\prime \prime} \eta-\frac{T_{0_{c}} B}{U_{c}^{\prime}}\left(1+l^{2}\right) \log \left(1+\eta^{2}\right)^{1 / 2}\right\} \mathcal{L}\left(x_{1}\right) \exp [i(\xi+\zeta)]
\end{aligned}
$$

and note that the second integral in $(29 d)$ is of $O\left(\eta^{-1}\right)$ for large $\eta$.

We now perform the asymptotic matching principle of Van Dyke in the form "2 main of ( 2 critical) $=2$ critical of $(2$ main)" for the streamwise velocity component. With the help of Appendix A. (29d), and (26b), we find

$$
\begin{gathered}
\hat{u}^{(1)}(0)=-\int_{0}^{ \pm \infty} Z(\eta) \mathrm{d} \eta-U_{\mathrm{c}}^{\prime} G_{ \pm}^{(1)}+\Lambda U_{\mathrm{c}}^{\prime}\left[q_{ \pm} y_{\mathrm{c}}-\frac{1+\ell^{2}}{q_{ \pm}} \int_{ \pm \infty}^{y_{\mathrm{c}}} J_{ \pm}(y) \mathrm{d} y\right] \\
\times \mathcal{L}\left(x_{1}\right) \exp [i(\xi+\zeta)]+R\left(\xi, \zeta, x_{1}\right),
\end{gathered}
$$

where $R$ is a nonessential remainder that is actually known from matching. but whose precise form is not needed for the determination of the amplitude equation. There is no ( \pm ) label on $R$. Recall that $G_{ \pm}^{(1)}$ is defined by $(17 d)\left(\mathscr{B}_{ \pm}\right.$in this equation may be obtained from $(23 a)$ in which we set $\mathscr{B}_{-}=0$; see earlier discussion) and $J_{ \pm}(y)$ is introduced in (A 4). 
We now have two equations for the single unknown $\hat{u}^{(1)}(0)$. This is because we may match the critical layer solution to the $( \pm)$ main layers. The existence of $\hat{u}^{(1)}(0)$ (i.e. solvability) requires

$$
\exp [-i(\xi+\zeta)] \int_{-\infty}^{\infty} Z(\eta) \mathrm{d} \eta=\nu_{1} \mathcal{L}^{\prime}\left(x_{1}\right)+\nu_{2} \mathcal{A}\left(x_{1}\right)
$$

where

$$
\begin{aligned}
v_{1} & =\Lambda U_{c} U_{c}^{\prime}\left\{\frac{1}{U_{-}-U_{c}}\left[1-\frac{1+\ell^{2} U_{-} / U_{c}}{q_{-}^{2}}\right]-\frac{1}{U_{+}-U_{c}}\left[1-\frac{1+\ell^{2} U_{+} / U_{c}}{q_{+}^{2}}\right]\right\}, \\
\nu_{2} & =\Lambda U_{c}^{\prime}\left\{\frac{1+\ell^{2}}{q_{-}} \int_{-\infty}^{y_{c}} J_{-}(y) \mathrm{d} y+\frac{1+\ell^{2}}{q_{+}} \int_{y_{c}}^{+\infty} J_{+}(y) \mathrm{d} y\right. \\
& \left.+q_{-} \int_{-\infty}^{y_{\mathrm{c}}}\left[\Gamma_{-}(y)-1\right] \mathrm{d} y+q_{+} \int_{y_{\mathrm{c}}}^{+\infty}\left[\Gamma_{+}(y)-1\right] \mathrm{d} y\right\} .
\end{aligned}
$$

and $\Gamma_{ \pm}(y)$ is defined in $(14 b)$.

We interpret $(3 \mid a)$ as the evolution (amplitude) equation for $\mathcal{A}\left(x_{1}\right)$. For a given mixing layer, the constants $\nu_{1}$ and $\nu_{2}$ may be calculated, and the integral of $Z$ may be obtained (most simply) as follows.

Since the desired (improper) integral of $Z$ exists in the Riemann sense, it also exists in the sense of the Cauchy principal value, $\lim _{b \rightarrow \infty} \int_{-b}^{b}=f_{-\infty}^{\infty}$. It is immaterial, in principle, which of these two procedures is used to calculate the value of this integral. However. from practical considerations, it is simpler to calculate the Cauchy principal value because the second term in the braces of $(29 b)$, being an odd function of $\eta$. integrates to zero. Thus.

$$
\int_{-\infty}^{\infty} Z(\eta) \mathrm{d} \eta=\int_{-\infty}^{\infty} \mathrm{Z}(\eta) \mathrm{d} \eta=\int_{-\infty}^{\infty}\left\{\frac{\partial \hat{u}^{(1)}}{\partial \eta}-i \Lambda U_{c}^{\prime \prime} \mathcal{L}\left(x_{1}\right) \exp [i(\xi+\zeta)]\right\} \mathrm{d} \eta
$$


After substituting (28c) into (32a) and using the identity

$$
\int_{-\infty}^{\infty} \eta^{k} W_{\mathrm{k}}\left(\eta, x_{1}\right) \mathrm{d} \eta=\frac{2 \pi k !}{(-i \alpha)^{\mathrm{k}}|\alpha|} \mathcal{L}\left(x_{1}\right) \int_{-\infty}^{0} \delta(x) \mathrm{d} x, \quad k=0,1,2, \ldots
$$

we find

$$
\begin{gathered}
\int_{-\infty}^{\infty}\left\{\frac{\partial \hat{u}^{(1)}}{\partial \eta}-i \Lambda U_{c}^{\prime \prime} \mathcal{C}\left(x_{1}\right) \exp [i(\xi+\zeta)]\right\} \mathrm{d} \eta=-\frac{\pi i}{|\alpha|} \frac{T_{0_{c}} B\left(1+\ell^{2}\right)}{U_{c}} \\
\times \mathcal{M}\left(x_{1}\right) \exp [i(\xi+\zeta)] .
\end{gathered}
$$

where we have assigned the (correct) value. $1 / 2$, to the integral of the delta function in $(32 b)$. Note that $(32 b)$ is a purely formal result that may be established by the use of generalized functions (Lighthill 1978); the two possible values for the integral of the delta function are 0 and 1 (whenever the upper limit takes on values $0^{-}$and $0^{+}$. respectively).

Although it would be tempting to conclude from these remarks that the value of the integral on the right-hand side of $(32 b)$ is $1 / 2$, such a conclusion can only be arrived at by a more careful procedure. This involves the ideas of "phase jump" associated with the logarithmic branches that arise in the stability of shear flows with critical layers (Maslowe 1981). As is well known, this phase jump depends on the algebraic sign of $\alpha$ $=U_{\mathrm{c}}^{\prime} / U_{\mathrm{c}}$, hence the presence of $|\alpha|$ in $(32 c)$. Our final remark deals with a generalization of $(32 b)$ that is also needed: When the exponent of $\eta$ is less than the value of $k$ on the subscript of $W_{\mathrm{k}}$, the integral vanishes. Therefore, only the first three terms on the right-hand side of (28c) yield a nonzero contribution.

If we now substitute $(32 c)$ into $(31 a)$, after invoking $(32 a)$, we indeed arrive at an amplitude equation for $\mathcal{L}\left(x_{1}\right)$.

In the nonlinear theory, which is developed in the following sections, the amplitude equation ( $31 a)$ suffers a modification. Briefly, the right-hand side remains unaltered, but the left-hand side will contain a term additional to that given in (32c). 


\section{Amplitude scaling for the nonlinear problem}

In our discussion so far, the characteristic amplitude $\epsilon \ll 1$ has not played a role because the problem has been linearized. We now establish the distinguished scaling. $\epsilon=\epsilon(\sigma)$. which will enable us to include the effects of nonlinearities in the phase jump condition, $(32 c)$.

Of course, as is seen from (1), there are many nonlinear terms in the equations of motion. In order to motivate our discussion and the derivation of the scaling, we shall use the convective nonlinearity, $\mathbf{u} \cdot \nabla \mathbf{u}$, as a typical term; specifically, we write the $x$ component of $(1 b)$,

$$
\frac{\mathrm{D} u}{\mathrm{D} t}+u \frac{\partial u}{\partial x}+\ldots=0
$$

and focus attention on the first two terms of $(33 a)$.

The nonlinear terms first show up in the critical layer because the linear term. $\mathrm{D} u / \mathrm{D} t$. is relatively small here. After using $(19 a)$ and $(3 c)$ in $(33 a)$, we find

$$
\sigma^{2}\left\{\mathscr{L}^{(0)}+\sigma \mathscr{L}^{(1)}+\ldots\right\} u+\sigma u \frac{\partial u}{\partial \xi}+\ldots=0 .
$$

Since, from $(20 a), u=O(\epsilon)$, the nonlinear "forcing" term in $(33 b)$ is $O\left(\sigma \epsilon^{2}\right)$ and the resultant first and zeroth harmonics are of $O\left(\sigma \epsilon^{2} / \sigma^{2}\right)=O\left(\epsilon^{2} / \sigma\right)$. Note that this is significantly larger than what would be obtained by simply squaring the fundamental (i.e. $O\left(\epsilon^{2}\right)$ ). A further nonlinear interaction of these harmonics with the fundamental of $O(\epsilon)$ produces nonlinear terms of $O\left(\sigma \epsilon \epsilon^{2} / \sigma\right)=O\left(\epsilon^{3}\right)$. It is at this level in the perturbation analysis where the fundamental reappears again for the first time due to nonlinearities, and, in order to affect the phase jump condition (hence the evolution equation for $\mathcal{H}\left(x_{1}\right)$ ), we require that the slow aging of the fundamental (on the scale $x_{1}$ ) also be present at this level. Thus, from $(33 b)$.

$$
\sigma^{2} \sigma \epsilon=O\left(\epsilon^{3}\right)
$$


or $\epsilon=O\left(\sigma^{3 / 2}\right)$. For convenience, we set $\epsilon=\sigma^{3 / 2}$ without loss of generality. This is our distinguished scaling.

It should be noted that this type of scaling argument was used by Hickernell (1984) in a geophysical problem and by Goldstein and his colleagues in a more closely related set of problems involving oblique modes near the neutral point (Goldstein \& Choi 1989. Goldstein \& Leib 1989) and resonant triad interactions (Goldstein \& Lee 1992).

It is somewhat difficult to make a direct comparison between our distinguished scaling and those of others because of the more elaborate triple-layer structure of the present problem (i.e. outer, main, and critical layers) as compared with the usual double-layer structure (i.e. outer and critical layers) found by previous investigators for their problems. Nevertheless, the following remarks may be helpful: let $\epsilon_{0} \ll 1$ be the characteristic magnitude of the instability mode in the outer layers. We see from (4) that $\epsilon_{0}=O(\epsilon \sigma)=O\left(\sigma^{5 / 2}\right)$ because of our distinguished scaling. Thus, $\sigma=O\left(\epsilon_{0}^{2 / 5}\right)$; when our result is interpreted in this way, it is reminiscent of the scaling used by Hickernell (1984) and Goldstein \& Leib (1989).

There remains to assess the importance of nonlinearities in the main and outer layers. By using (11) and $(10 a)$ in $(33 a)$, we find that the nonlinearities do not enter in the main layers to the required order of accuracy. Similar remarks hold for the outer layers. Therefore, with the exception of the critical layer, the other layers are governed by linear dynamics. Thus, the relevant solutions for the fundamental disturbance are essentially available in $\$ 3$ and $\$ 4$.

Based on the above remarks and (20), we find that the perturbation quantities in the critical layer expand as

$$
u=\sigma^{3 / 2}\left(\hat{u}^{(0)}+\sigma^{1 / 2} \hat{u}^{(1 / 2)}+\sigma \hat{u}^{(1)}+\ldots\right),
$$

with similar expressions for $w, \rho$, and $T$, whereas 


$$
p=\sigma^{5 / 2}\left(\hat{p}^{(0)}+\sigma^{1 / 2} \hat{p}^{(1 / 2)}+\sigma \hat{p}^{(1)}+\ldots\right)
$$

and

$$
v=\sigma^{7 / 2}\left(\hat{\vartheta}^{(0)}+\sigma^{1 / 2} \hat{v}^{(1 / 2)}+\sigma \hat{\vartheta}^{(1)}+\ldots\right)
$$

where the $\hat{(}^{(1 / 2)}$ terms are inserted in order to accommodate the zeroth and first harmonics (which are, for example, in the $u$-equation of $O\left(\epsilon^{2} / \sigma\right)=O\left(\sigma^{2}\right)$ ). The nonlinear part of the complete substantial derivative, to the accuracy required herein, becomes

$$
\mathbf{u} \cdot \nabla=\sigma^{s / 2}\left[N^{(0)}+\sigma^{1 / 2} N^{(1 / 2)}+O(\sigma)\right]
$$

where

$$
N^{(j)}=\hat{u}^{(j)} \frac{\partial}{\partial \xi}+\hat{\hat{\sigma}}(\mathrm{j}) \frac{\partial}{\partial \eta}+l \hat{w}^{(j)} \frac{\partial}{\partial \zeta}, \quad j=0,1 / 2 .
$$

After substituting (34) and (35) into (1) and expanding the base flow in Taylor series about the critical point, we obtain a hierarchy of equations for $(\hat{\cdot})^{(j)}, j=0,1 / 2,1$. The lowest-order solutions, $\hat{(}^{(0)}$, are essentially given by linearized theory; these were already obtained in $\$ 5$ using complex representation. For the nonlinear problem, we consider two oblique modes, with spanwise wavenumbers of $( \pm \ell)$ and exponential factors of $\exp [i(\xi \pm \xi)]$, respectively. The complex amplitudes of these modes remain $\mathcal{M}\left(x_{1}\right)$. although it is possible to generalize our analysis to unequal amplitudes.

After superposing the oblique modes and using the imaginary part of this complex quantity to represent the physically real solution. we find from $(22 a)$. (24a), (26b), and $(26 c)$,

$$
\begin{aligned}
& \hat{p}^{(0)}=\gamma M^{2} \operatorname{Re}[i \mathcal{L} \exp (i \xi)] \cos \zeta . \\
& \hat{w}^{(0)}=\frac{\ell_{0_{c}}}{U_{\mathrm{c}}} \operatorname{Re}\left[i W_{0} \exp (i \xi)\right] \sin \zeta, \\
& \hat{u}^{(0)}=-\frac{\ell^{2} T_{0_{\mathrm{c}}}}{U_{\mathrm{c}}} \operatorname{Re}\left[W_{0} \exp (i \xi)\right] \cos \zeta-\Lambda U_{\mathrm{c}}^{\prime} \operatorname{Re}[\mathcal{L} \exp (i \xi)] \cos \zeta .
\end{aligned}
$$




$$
\begin{aligned}
\hat{\sigma}^{(0)}= & \frac{T_{0_{c}}\left(1+\ell^{2}\right)}{U_{c}^{\prime}} \operatorname{Re}[\mathcal{L} \exp (i \xi)] \cos \zeta \\
& +\Lambda U_{\mathrm{c}} \operatorname{Re}\left[\mathcal{L}^{\prime} \exp (i \xi)+i \alpha \eta \mathcal{L} \exp (i \xi)\right] \cos \zeta .
\end{aligned}
$$

where $\operatorname{Re}(\cdot)$ denotes the real part of a complex number, $\mathcal{L}=\mathcal{A}\left(x_{1}\right), \mathcal{L}=\mathrm{d} \mathscr{L}\left(x_{1}\right) / \mathrm{d} x_{1}$. and $W_{0}=W_{0}\left(\eta, x_{1}\right)$. In order to simplify the notation a bit, we make use of the convention that, whenever the argument of a function on the right-hand side of an equation is not shown explicitly, it is understood to be the usual field variables $x_{1}$ or $\left(\eta, x_{1}\right)$, etc. For example, $I_{n}\left(\eta, x_{1}\right)$ will be written as $I_{n}(n=0,1, \ldots)$.

It should be noted that, if we artificially set the vortex-sheet displacement parameter. $\Lambda$, to zero in (36), we recover (albeit in the current notation) the critical layer solutions used by others (e.g. Goldstein \& Lee 1992). This comparison points to the considerable algebraic complexity that we will encounter in the treatment of the nonlinear problem. In order to keep this complexity at a minimum. we shall focus on the new terms that come about because $\Lambda \neq 0$ (see Appendix B). We might point out that, because of the presence of these terms. the cross-stream velocity component, $\hat{v}(0)$, is no longer a "constant" in the critical layer but varies linearly with $\eta$ (see (36d)).

\section{Nonlinear solutions in the critical layer}

Although the linearized theory suggests that we should solve for $\partial \hat{u}^{(1)} / \partial \eta$ and integrate this quantity from $\eta=-\infty$ to $\eta=+\infty$ in order to find the logarithmic phase jump (see $(32 c)$ ), the analysis of Goldstein \& Lee (1992) clearly indicates that a significant economy in algebraic manipulations is brought about in the nonlinear case by introducing the new variable

$$
Q=\frac{\partial \hat{u}^{(1)}}{\partial \eta}+l \frac{\partial \hat{w}^{(1)}}{\partial \eta}
$$

whose fundamental Fourier component. $Q_{1,1}$, is defined by 


$$
Q=Q_{1,1} \exp [i(\xi+\zeta)]+\ldots
$$

and obeys

$$
U_{\mathrm{c}}\left[\frac{\partial}{\partial x_{1}}+i \alpha \eta\right] Q_{1,1}=-\frac{\partial \mathcal{N}_{11}}{\partial \eta}+(\text { linear terms })_{1,1}
$$

The nonlinear term, $\mathcal{N}$, in $(37 c)$ arises from the convective nonlinearities in the equations of motion. It is defined by

$$
\mathcal{N}=N^{(0)}\left(\hat{u}^{(1 / 2)}+l \hat{w}^{(1 / 2)}\right)+N^{(1 / 2)}\left(\hat{u}^{(0)}+l \hat{w}^{(0)}\right)
$$

with $\mathcal{N}_{1,1}$ denoting its fundamental component,

$$
\mathcal{N}=\mathscr{N}_{1,1} \exp [i(\xi+\zeta)]+\ldots
$$

where the ellipses in $(37 b, e)$ stand for the other Fourier components. Recall $(35 b)$ for the definitions of $N^{(0)}$ and $N^{(1 / 2)}$.

We note that the derivation of $(37 c)$ is straightforward by taking linear combinations of the equations for $\partial \hat{u}^{(1)} / \partial \eta$ and $\partial \hat{w}^{(1)} / \partial \eta$. observing that $\partial \hat{p}^{(j)} / \partial \eta=0$ $(j=0,1 / 2,1)$, and invoking the pressure form of the equation of continuity (i.e. the energy equation) to eliminate $\left(\partial \hat{u}^{(1)} / \partial \xi+\partial \hat{v}^{(1)} / \partial \eta\right)$ in favor of $\left[-\left(\ell \partial \hat{w}(1) / \partial \zeta+\partial \hat{u}^{(0)} / \partial x_{1}\right)\right]$.

The phase jump for the nonlinear problem is obtained most easily by using $Q_{1,1} \exp [i(\xi+\zeta)]$ in place of $\partial \hat{u}^{(1)} / \partial \eta$ in $(32 c)$. This is permissible since the fundamental component of $\widehat{w}^{(1)}$ has no jump across the critical layer; a fact guaranteed by the behavior of the main-layer solutions at the edges of the critical layer (see (A 7)).

Since $Q_{1,1}$ obeys a linear, inhomogeneous equation, the phase jump associated with $Q_{1,1}$ is the superposition of the effects of the two forcing terms in (37c). Insofar as the "linear terms" in this equation can be easily obtained from (27a) and (28a), and the phase jump associated with these terms has been calculated already, we shall not say more about these terms. Our principal focus is to calculate the effect of the nonlinear forcing term $\mathscr{N}_{1,1}$ 
Before we go any further with some general results, it is advantageous to introduce the complex Fourier-series representation of a perturbation variable,

$$
\hat{f}^{(j)}\left(\xi . \eta, \zeta . x_{1}\right)=\sum_{m} \sum_{n} \hat{F}_{\mathrm{n}, \mathrm{m}}^{(j)}\left(\eta, x_{1}\right) \exp [i(n \xi+m \xi)], \quad j=0,1 / 2,
$$

where $f$ may stand for $u, v, w$, etc. Of course, some of the $F^{\prime} s$ may vanish. Note that for a function $\hat{f}^{(j)}$ the Fourier coefficients are denoted by $\hat{F}_{\mathrm{n}, \mathrm{m}}^{(j)}$. For example, if $j=0$ and $f \rightarrow v$, we find from (38) and (36d)

$$
\begin{gathered}
\hat{V}_{1.1}^{(0)}=\hat{V}_{1,-1}^{(0)}=\mathscr{G} . \\
\hat{V}_{-1,-1}^{(0)}=\hat{V}_{-1,1}^{(0)}=\tilde{G} .
\end{gathered}
$$

where

$$
G=\frac{T_{0_{c}}\left(1+\ell^{2}\right)}{4 U_{\mathrm{c}}^{\prime}} \mathcal{A}+\frac{\Lambda U_{\mathrm{c}}}{4}\left(\mathcal{L}^{\prime}+i \alpha \eta \mathcal{L}\right)
$$

and $\tilde{(\cdot)}$ denotes the complex conjugate of $(\cdot)$. All other $\hat{V}_{n, m}^{(0)}$ vanish. Similar expressions hold for $\hat{U}_{\mathrm{n}, \mathrm{m}}^{(0)}$ and $\hat{W}_{\mathrm{n}, \mathrm{m}}^{(0)}$, which are derivable from $(36 c, b)$ and (38).

In order to calculate the nonlinear forcing term, $\partial\left(\mathcal{N}_{1,1}\right) / \partial \eta$. we carry out the operations indicated in $(37 d)$ by using the Fourier-series representation of the variables. The resultant expression may be simplified slightly by employing the equations

$$
\begin{gathered}
\hat{W}_{0,0}^{(1 / 2)}=\hat{W}_{2,0}^{(1 / 2)}=0 \\
\hat{U}_{2.2}^{(1 / 2)}+\ell \hat{W}_{2,2}^{(1 / 2)}=0
\end{gathered}
$$

and the expressions for the Fourier components of $\hat{(\cdot)^{(0)}}$, as obtained from (36). Note that the validity of $(40 a, b)$ is discussed in Appendix B. After a modest amount of algebra, we find

$$
\frac{\partial \mathcal{N}_{1,1}}{\partial \eta}=\sum_{k=1}^{7} T_{k}
$$


where

$$
\begin{gathered}
T_{1}=\mathcal{G} \frac{\partial^{2} \hat{U}_{0.0}^{(1 / 2)}}{\partial \eta^{2}}, \quad T_{2}=\tilde{G} \frac{\partial^{2} \hat{U}_{2,0}^{(1 / 2)}}{\partial \eta^{2}}, \\
T_{3}=\mathscr{G} \frac{\partial^{2}}{\partial \eta^{2}}\left[\hat{U}_{0.2}^{(1 / 2)}+\ell \hat{W}_{0.2}^{(1 / 2)}\right], \\
T_{4}=-\frac{\ell^{2} T_{0}}{2 U_{c}} \frac{\partial}{\partial \eta}\left[\hat{V}_{2,0}^{(1 / 2)} \frac{\partial \tilde{W}_{0}}{\partial \eta}\right] . \\
T_{5}=-\frac{\ell^{2} T_{0}}{U_{c}} \frac{\partial}{\partial \eta}\left[i \hat{U}_{0.2}^{(1 / 2)} W_{0}+\frac{1}{2} \hat{V}_{0.2}^{(1 / 2)} \frac{\partial W_{0}}{\partial \eta}\right], \\
T_{6}=-\frac{i \Lambda U_{c}^{\prime}}{2} \tilde{\mathcal{L}} \frac{\partial \hat{U}_{2,0}^{(1 / 2)}}{\partial \eta}, \\
T_{7}=\frac{i \Lambda U_{c}^{\prime} \ell}{2} \mathcal{L} \frac{\partial \hat{W}_{0.2}^{(1 / 2)}}{\partial \eta},
\end{gathered}
$$

and $W_{0}$ is defined by $(24 b)$. This particular grouping of the terms is helpful in reducing the subsequent algebra (Lee 1992, private communication); they represent the relevant "cubic" nonlinear interactions that produce the fundamental.

Unfortunately the algebraic manipulations do become extremely tedious beyond this point--we shall be content with presenting a few of the key steps in Appendix B. However. the details of these manipulations may be found in the dissertation by Gartside (1995).

The overall plan is to solve for the $(\hat{\cdot})^{(1 / 2)}$ variables in the critical layer (see (34)). These are forced by the quadratic interactions of the fundamental with itself; the solution provides the Fourier coefficients (e.g. $\hat{U}_{0.0}^{(1 / 2)}$, etc.) needed in $(41 b-h)$. We then solve (37c) to obtain the seven contributions to $Q_{1,1}$. say $Q_{1,1}^{(\mathrm{k})}$, arising from each of the nonlinear forcing terms $T_{\mathrm{k}}(k-1, \ldots, 7)$. The nonlinear correction to the phase jump condition. (32c), may be found according to our previous discussion in this section. For the purposes of illustration, some of the algebraic details are carried out in Appendix C 
for the simplest of the terms, namely, $T_{7}$. The other terms may be handled in a similar fashion, as shown by Gartside (1995).

After a considerable amount of algebra, we find that the total nonlinear correction to the phase-jump is

$$
\begin{aligned}
\int_{-\infty}^{\infty} Q_{1,1}\left(\eta, x_{1}\right) \mathrm{d} \eta & =-\frac{i \pi T_{0_{c}}^{3} \sin ^{2} \theta \cos 2 \theta}{16 U_{c}^{5} \cos ^{6} \theta} \operatorname{sgn}\left(U_{c}^{\prime}\right) \int_{-\infty}^{x_{1}} \mathcal{M}\left(\hat{x}_{1}\right) \mathrm{d} \hat{x}_{1} \int_{-\infty}^{\hat{x}_{1}} \hat{Z}\{\hat{Z}(\hat{Z}+\mathcal{Z}) \\
& \left.+2 \mathcal{Z}(\mathcal{Z}-\hat{Z}) \sin ^{2} \theta\right\} \mathcal{M}\left(\hat{x}_{1}\right) \tilde{\mathcal{L}}\left(\hat{x}_{1}+\hat{x}_{1}-x_{1}\right) \mathrm{d} \hat{x}_{1} .
\end{aligned}
$$

and

$$
\begin{gathered}
\tan \theta=\ell, \\
\hat{Z}=\hat{x}_{1}-x_{1} . \\
\hat{Z}=\hat{x}_{1}-x_{1} .
\end{gathered}
$$

It is assumed that $U_{\mathrm{c}}>0$ in these equations. We multiply $(42 a)$ by $-4 i \exp [i(\xi+\zeta)]$ and add this to the right-hand side of $(32 c)$ in order to obtain the total phase jump (due to linear and nonlinear effects) to be used in the amplitude equation (31a). We shall give the canonical form of this equation and the corresponding initial conditions (as $x_{1} \rightarrow-\infty$ ) in the next section, after introducing a series of transformations in order to reduce the number of parameters.

\section{Discussion and conclusions}

In order to present the simplest evolution equation for the amplitude, we introduce scale transformations for the independent and dependent variables:

$$
\begin{gathered}
\bar{x}=-\frac{v_{2} x_{1}}{v_{1}}, \quad v_{2} / v_{1}<0, \\
\mathcal{M}\left(x_{1}\right)=\Gamma A(\bar{x}) .
\end{gathered}
$$


where $v_{1}$ and $v_{2}$ are defined by $(31 b, c)$ and $\Gamma$ is a complex constant. Recall that $v_{1}$ and $v_{2}$ are determined from the base flow profiles and the underlying linear instability theory that defines the critical point. The magnitude of $\Gamma$ (i.e. $|\Gamma|)$ is chosen such that the absolute value of the coefficient in front of the integral on the right-hand side of (42a) becomes unity and the phase of $\Gamma$, together with a shift in the origin of the slow variable $\vec{x}$, is used to eliminate a factor of proportionality from the upstream boundary condition. Similar transformations have been used by Goldstein and his colleagues (e.g., Goldstein \& Lee 1992); the algebraic details are quite straightforward and will not be repeated here.

Using these transformations, as well as $(32 c)$ and $(42 a, b)$, we find that the amplitude equation becomes

$$
\begin{aligned}
\frac{\mathrm{d} A}{\mathrm{~d} x_{1}}-i K A= & \operatorname{sgn}\left(v_{2}\right) \operatorname{sgn}\left(U_{\mathrm{c}}^{*}\right) \operatorname{sgn}(\cos 2 \theta) \int_{-\infty}^{x_{1}} A\left(\hat{x}_{1}\right) \mathrm{d} \hat{x}_{1} \\
& \times \int_{-\infty}^{\hat{x}_{1}} \hat{Z}\left\{\hat{Z}(\hat{Z}+\hat{Z})+2 \hat{Z}(\hat{Z}-\hat{Z}) \sin ^{2} \theta\right\} A\left(\hat{x}_{1}\right) \tilde{A}\left(\hat{x}_{1}+\hat{x}_{1}-x_{1}\right) \mathrm{d} \hat{x}_{1},
\end{aligned}
$$

where we have renamed our new slow variable (i.e. (43a) and a shift in the origin) to be $x_{1}$ in order to make use of convenient definitions $(42 c, d)$ and to keep the notation simple. However, the presence of $A$ (rather than $\mathcal{C}$ ) in (44) serves as a reminder that this equation is written in terms of the "scaled" variables. The upstream condition associated with (44) is

$$
A \rightarrow e^{i \mathrm{~K} x_{1}} \quad \text { as } x_{1} \rightarrow-\infty \text {. }
$$

where

$$
K=-\frac{\pi \operatorname{sgn}\left(U_{c}^{\prime}\right)}{v_{2} \cos ^{2} \theta}\left(\frac{T_{0_{c}} U_{c}^{\prime \prime}}{U_{c}^{\prime 2}}\right)\left(1-\frac{T_{0_{c}}^{\prime} U_{c}^{\prime}}{T_{0_{c}} U_{c}^{\prime \prime}}\right)-i
$$

This condition expresses the fact that very far upstream the base flow is perturbed by a linear instability mode. 
The solution to the amplitude equation, (44), formally depends on three parameters: $\operatorname{Re}(K)$, the coefficient in front of the double integral, and $\theta=\tan ^{-1} \ell$. We shall study (numerically) the behavior of $A\left(x_{1}\right)$ as a function of these parameters; some representative results are presented below.

In figures 3 and 4 we show representative results for $\operatorname{Re}(K)=K_{\mathrm{R}}$ as a function of Mach number, $m=\Delta U M$, for three different temperature ratios. $T_{0_{-}} / T_{0_{+}}=T_{0_{-}}$. The velocity profile in the mixing layer is given by

$$
U\left(y_{H}\right)-U_{m}+\frac{\Delta U}{2} \tanh \left(y_{H}\right)
$$

where

$$
U_{\mathrm{m}}=\frac{U_{+}+U_{-}}{2}, \quad \Delta U=U_{+}-U_{-}>0
$$

and the temperature profile is specified by Crocco's relation

$$
T_{0}\left(y_{\mathbf{H}}\right)=1-\frac{1-T_{0}}{2}\left[1-\tanh \left(y_{\mathrm{H}}\right)\right]+\frac{\gamma-1}{8} m^{2}\left[1-\tanh ^{2}\left(y_{\mathrm{H}}\right)\right]
$$

where $y_{\mathrm{H}}$ is the Howarth-Dorodnitsyn variable. It is related to the "physical" crossstream coordinate, $y$, by the transformation

$$
y=\int_{0}^{y_{\mathrm{H}}} T_{0}(\eta) \mathrm{d} \eta
$$

These profiles were also used by Jackson \& Grosch (1989) in their study of the linear stability of compressible mixing layers.

It is seen from figures 3 and 4 that, generally, $K_{\mathrm{R}}$, is of order unity and varies roughly linearly with $m$, for $m$ sufficiently large. The fast and slow modes are defined by the inequalities $U_{\mathrm{c}}>U_{\mathrm{m}}$ and $U_{\mathrm{c}}<U_{\mathrm{m}}$, respectively. where $U_{\mathrm{c}}$ is the convection speed of the instability mode as obtained from the vortex-sheet dispersion relation, $(7 a)$. Note that when the temperature ratio is unity, the two curves for $K_{\mathrm{R}}$ are mirror images of each other about the horizontal coordinate axis. This is because $\left(U-U_{\mathrm{m}}\right)$ is an odd function of $y=y_{\mathrm{H}}$. 
Interestingly enough, the solution of the nonlinear amplitude equation, (44), does not depend on $K_{\mathrm{R}}$ in an essential way. To see this, consider the trial solution

$$
A=|A| e^{\mathrm{i} \mathrm{K}_{\mathrm{R}} \mathrm{x}_{1}}
$$

which assumes that the phase of the complex amplitude is linear in $x_{1}$. After substituting (47) into (44) and simplifying. we find

$$
\begin{aligned}
\frac{d|A|}{\mathrm{d} x_{1}}-|A|= & \operatorname{sgn}\left(\nu_{2}\right) \operatorname{sgn}\left(U_{c}^{\prime}\right) \operatorname{sgn}(\cos 2 \theta) \int_{-\infty}^{0}\left|A\left(\hat{Z}+x_{1}\right)\right| \mathrm{d} \hat{Z} \\
& \times \int_{-\infty}^{\hat{Z}} \mathscr{R}(\hat{Z} \cdot \hat{Z})\left|A\left(\hat{Z}+x_{1}\right)\right|\left|A\left(\hat{Z}+\hat{Z}+x_{1}\right)\right| \mathrm{d} \hat{Z},
\end{aligned}
$$

where the kernel, $\mathscr{K}(\hat{Z}, \hat{Z})$ is defined by

$$
\mathscr{K}(\hat{Z}, \hat{Z})=\hat{Z}\left\{\hat{Z}(\hat{Z}+\hat{Z})+2 \hat{Z}(\hat{Z}-\hat{Z}) \sin ^{2} \theta\right\}
$$

This nonlinear equation for $|A|$ is independent of $K_{\mathrm{R}}$.

Furthermore. for our mixing layer, $U_{\mathrm{c}}^{\prime}>0$ and, for profiles given by equation (46). $v_{2}$ turns out to be negative (as obtained from numerical evaluations of $(3 \mid c)$ ). Thus the coefficient in front of the double integral in $(48 a)$ is $\mp 1$ according to $\theta \lessgtr \pi / 4$. The algebraic kernel in (48) is negative over the domain of integration.

Thus, for $\theta<\pi / 4$, the amplitude of the oblique mode monotonically increases with coordinate $x_{1}$. This observation is confirmed in figure 5 ; the corresponding phase of $A$ is shown in figure 6. The latter figure establishes the validity of the trial solution. (47), in this particular case.

The numerical result in figure 5 suggests that $\left|A\left(x_{1}\right)\right|$ develops a singularity at some finite value of $x_{1}$, say $x_{1}^{(s)}$. This observation is consistent with the findings of other investigators (Goldstein \& Choi 1989, Goldstein \& Leib 1989); indeed, near the singularity, the dominant terms in (48a) can be balanced by a solution of the form 


$$
\left|A\left(x_{1}\right)\right| \sim \frac{1}{\left[x_{1}^{(s)}-x_{1}\right]^{3}}
$$

as $x_{1} \rightarrow x_{1}^{(s)}$ from below.

In order to establish the presence of a singularity when $\theta<\pi / 4$, consider the inequality

$$
\begin{gathered}
-\int_{-\infty}^{0}\left|A\left(\hat{Z}+x_{1}\right)\right| \mathrm{d} \hat{Z} \int_{-\infty}^{\hat{Z}} \mathscr{K}(\hat{Z} . \hat{Z})\left|A\left(\hat{Z}+x_{1}\right)\right|\left|A\left(\hat{Z}+\hat{Z}+x_{1}\right)\right| \mathrm{d} \hat{Z} \\
\quad z-\int_{-\infty}^{0} \mathrm{~d} \hat{Z} \int_{-\infty}^{\hat{Z}} \mathscr{K}(\hat{Z}, \hat{Z})\left|A\left(\hat{Z}+\hat{Z}+x_{1}\right)\right|^{3} \mathrm{~d} \hat{Z} .
\end{gathered}
$$

which holds because $|A|$ is a monotonically increasing function, and $\hat{Z} \leq 0$ and $\hat{Z} \leq 0$ over the domain of integration. Both sides of $(50)$ are positive. The integral on the right-hand side of (50) may be reduced to a one-dimensional integral after the introduction of new variables of integration; the final result is

$$
\Omega \int_{0}^{\infty} \xi^{4}\left|A\left(x_{1}-\xi\right)\right|^{3} \mathrm{~d} \xi,
$$

where

$$
\Omega=\frac{1}{4 !}\left[1+\frac{3 \sin ^{2} \theta}{2}\right]>0
$$

Motivated by the discussion above, we introduce the comparison equation

$$
\frac{\mathrm{d} g}{\mathrm{~d} x_{1}}-g=\Omega \int_{0}^{\infty} \xi^{4} g^{3}\left(x_{1}-\xi\right) \mathrm{d} \xi
$$

with upstream boundary condition

$$
g \rightarrow e^{x_{1}} \quad \text { as } \quad x_{1} \rightarrow-\infty,
$$

for the positive function $g=g\left(x_{1}\right)$. This function serves as a lower bound for $\left|A\left(x_{1}\right)\right|$; 
$\left|A\left(x_{1}\right)\right| \geq g\left(x_{1}\right)$. The formal solution of (52) is

$$
g\left(x_{1}\right)=e^{x_{1}} \sum_{n=0}^{\infty} a_{n} e^{2 n x_{1}}
$$

where, for $n=0,1,2, \ldots$

$$
a_{\mathrm{n}+1}=\frac{4 ! \Omega}{2^{6}} \frac{1}{(n+1)(n+3 / 2)^{5}} \sum_{m=0}^{n} \sum_{r=0}^{n-m} a_{\mathrm{m}} a_{\mathrm{r}} a_{\mathrm{n}-\mathrm{m}-\mathrm{r}}
$$

with $a_{0}=1$. It is found numerically that the "tail end" of (53a) is asymptotic to a power series whose radius of convergence, $x_{1}^{(r)}$, can be obtained from the use of the ratio test as

$$
x_{1}^{(r)}=2.91-\frac{1}{2} \log \Omega
$$

Since for $x_{1}>0,(53 a)$ is a series with positive terms, $g$ must become singular at $x_{1}=$ $x_{1}^{(\mathrm{r})}$. Therefore, $\left|A\left(x_{1}\right)\right|$ must become singular at a finite value of $x_{1}=x_{1}^{(\mathrm{s})} \leq x_{1}^{(\mathrm{r})}$ in view of the fact that $|A| \geq 8$.

It is easy to show that the solution to the classical Landau equation.

$$
\frac{\mathrm{d} h}{\mathrm{~d} x_{1}}-h=\Omega h^{3}
$$

with upstream condition

$$
h \rightarrow e^{x_{1}} \quad \text { as } \quad x_{1} \rightarrow-\infty \text {. }
$$

does become singular at

$$
x_{1}=-\frac{1}{2} \log \Omega
$$

Evidently, the averaging of the nonlinear term. as implied by $(52 a)$ and $(48 a)$, does not eliminate the singularity, but merely shifts it downstream.

Representative numerical results for $\theta>\pi / 4$ are presented in figures 7 and 8 . It is seen from figure 8 that a sectionally linear phase is also consistent with nonlinear amplitude equation (44); this observation provides a generalization of trial solution (47). 
Although the behavior of the magnitude of the amplitude (figure 7) appears complicated, an explanation for this behavior is quite simple. It is based on the fact that the nonlinear term in (44) depends on the entire history of the amplitude and not only on its value at the current location.

For $x_{1}$ less than about 1.85. equation (48a) for $|A|$ is applicable since the phase is linear. From this equation, we see that the effect of the nonlinear integral is stabilizing because $\operatorname{sgn}(\cos 2 \theta)<0$. As a result, $A$ diminishes to zero (at $x_{1} \cong 1.85$ ) but $\mathrm{d} A / \mathrm{d} x_{1}$ is nonzero since the latter is balanced by the nonlinear integral that takes into account the entire evolution history of the complex amplitude. Hence, $A$ will smoothly pass through the origin in the complex plane, thereby suffering a phase jump of $\pm \pi$. For $x_{1}$ somewhat larger than 1.85. the effect of this phase jump is to render the coefficient of $\mathrm{d}|A| / \mathrm{d} x_{1}$ in (48a) negative or, equivalently, to make the nonlinear terms destabilizing.

As $x_{1}$ increases substantially from its value at the first spike $(\approx 1.85)$, the effect of the initial phase jump begins to show up in the calculation of the nonlinear integral. When this occurs to a significant extent, the effect of the phase jump cancels out between the cubic terms and the linear terms, and the nonlinear integral is again stabilizing. Thereby the second spike (or phase jump) near $x_{1} \cong 3.05$ is generated. This entire "quasi-periodic" scenario, in which the nonlinear terms are stabilizing or destabilizing over certain intervals of the $x_{1}$-axis, results in the successive spikes of figure 7 and in the phase jumps shown in figure 8 . The overall trend is a substantial increase in the magnitude of $A$ (say, for $x_{1}<4.5$ ), although there are narrow regions in the streamwise direction in which the amplitude may be small. This streamwise modulation of the disturbance is possible since the nonlinear interaction occurs in the form of an integral that is sensitive to upstream values of the amplitude.

We emphasize that the solutions for the complex amplitude were obtained by solving (44) numerically; trial solution (47) and equation (48a) for $|A|$ provide a convenient framework for the interpretation of the numerical results. 
Finally, when $\theta=\pi / 4$, the lowest-order nonlinear terms, as given by $(42 a)$, vanish. In this case, the nonlinear interaction between two oblique modes occurs at higher order in the small amplitude parameter. In order to understand this result, it is necessary to recall that (in linear theory for a slightly unstable mode) the velocity component of an oblique mode normal to the wave front is finite. whereas the component along the wave front is singular (in the critical layer).

When $\theta=\pi / 4$, the included angle between the oblique modes is $\pi / 2$. so that the component of the fluid velocity normal to one family of wave fronts will be completely dominated by the tangential velocity along the second family of wave fronts. Therefore, the nonlinear interaction between these modes is expected to be weaker than in the general case when both waves contribute (roughly equally) to the tangential velocity component along the wave fronts.

To summarize, the nonlinear interaction between two oblique modes in a supersonic mixing layer is governed by a nonlinear integro-differential equation of the HickernellGoldstein type. For each propagation angle, $\theta$, the magnitude of the scaled amplitude, $|A|$, is given by a universal curve (in other words, the only essential similarity parameter is $\theta$ ). When $\theta<\pi / 4$, the disturbance undergoes a monotonic and sudden increase in amplitude, which terminates in a singularity at a finite streamwise location. On the other hand, for $\theta>\pi / 4$, the streamwise development of $|A|$ is characterized by a series of modulations arising from the dual nature of the nonlinearity, which can be stabilizing or destabilizing, depending on the streamwise position.

The authors are grateful for the financial support provided by the NASA Lewis Research Center under grant NAG3-781. One of us (TFB) has benefited from stimulating discussions with Drs. M. E. Goldstein. L. Hultgren, and S. S. Lee on several aspects of this problem. The help of Sang Soo Lee, who provided some of his handwritten notes and a computer code, is deeply appreciated. Both items were used to provide an independent check on the validity of the results presented herein. 
Appendix A. Behavior of the solution in the main layers at the edge of the critical layer

The solution in the two main layers (denoted by \pm ) is given in $\$ 4$. In order to derive the behavior of this solution when $\left(y-y_{c}\right)$ is small, we first derive an approximation for the integral involving $\left(\Gamma_{ \pm}\right)^{-1}$. (see $(17 b)$ ). By Taylor series, for $\chi=y-$ $y_{\mathrm{c}}$ small, from $(14 b)$,

$$
\frac{1}{\Gamma_{ \pm}(y)}=C_{ \pm}\left[\frac{1}{\chi^{2}}+\frac{B}{\chi}+O(1)\right]
$$

where

$$
C_{ \pm}=\frac{\left(U_{ \pm}-U_{c}\right)^{2}}{T_{0_{ \pm}}} \frac{T_{0_{c}}}{U_{c}^{\prime 2}}
$$

and

$$
B=\left[\frac{T_{0}^{\prime}}{T_{0}}-\frac{U^{\prime \prime}}{U^{\prime}}\right]_{\mathrm{y}-\mathrm{y}_{\mathrm{c}}}
$$

If $B$ vanishes, the generalized inflection point criterion of Lees \& Lin (1946) is satisfied. We assume that $1 / \Gamma_{ \pm}$is analytic at all $y \neq y_{\mathrm{c}}$.

The above remarks imply that

$$
J_{ \pm}(y)=\frac{1}{\Gamma_{ \pm}(y)}-1-C_{ \pm}\left[\frac{1}{\chi^{2}}+\frac{B}{\chi\left(1+\chi^{2}\right)}\right]
$$

is an analytic function for all $y$ and is integrable at $y= \pm \infty$. The denominator under $B$ has been chosen with this objective in mind. Thus,

$$
\int_{ \pm \infty}^{y}\left[\frac{1}{\Gamma_{ \pm}(y)}-1\right] \mathrm{d} y=\int_{ \pm \infty}^{y_{\mathrm{c}}} J_{ \pm}(y) \mathrm{d} y+\int_{y_{c}}^{y} J_{ \pm}(y) \mathrm{d} y+C_{ \pm}\left[-\frac{1}{\chi}+B \log \frac{|x|}{\left(1+\chi^{2}\right)^{1 / 2}}\right] .
$$

In view of these preparatory remarks, the two-term critical layer expansion of the two-term main solution follows from (12), (17), and (A 5): 


$$
\begin{aligned}
\left\{\hat{u}^{(0)}+\sigma \hat{u}^{(1)}\right\} \exp [-i(\xi+\zeta)] \rightarrow i \Lambda\left(U_{c}^{\prime}+\sigma \eta U_{c}^{\prime \prime}\right) \mathcal{L}\left(x_{1}\right) \\
+\sigma \Lambda U_{c}^{\prime}\left\{-\mathcal{L}^{\prime}\left(x_{1}\right)+\left[q_{ \pm} y_{c}-\frac{1+\ell^{2}}{q_{ \pm}} \int_{ \pm \infty}^{y_{c}} J_{ \pm}(y) \mathrm{d} y\right] \mathcal{L}\left(x_{1}\right)\right\} \\
+\left\{\frac{\ell^{2} T_{0_{c}}}{U_{c}^{\prime} \eta}-\sigma\left(1+\ell^{2}\right) \frac{T_{0_{c}} B}{U_{c}^{\prime}} \log |\sigma \eta|+\frac{\sigma T_{0_{c}} U_{c}^{\prime \prime}}{U_{c}^{\prime 2}}\left[\frac{3}{2}+\ell^{2}\right]-\frac{\sigma T_{0_{c}}^{\prime}}{U_{c}^{\prime}}\right\} \mathcal{L}\left(x_{1}\right) \\
\quad-\sigma U_{c}^{\prime} G_{ \pm}^{(I)} \exp [-i(\xi+\zeta)],
\end{aligned}
$$

where $G_{ \pm}^{(1)}$ is defined by $(17 d)$. Similarly,

$$
\begin{aligned}
& \left\{\sigma \hat{w}^{(0)}+\sigma^{2} \hat{w}^{(1)}\right\} \exp [-i(\xi+\zeta)] \rightarrow-\frac{\ell}{U_{c}^{\prime} \eta}\left[T_{0_{c}}+\sigma T_{0_{c}}^{\prime} \eta-\frac{\sigma T_{0_{c}} U_{c}^{\prime \prime} \eta}{2 U_{c}^{\prime}}\right] \mathcal{H}\left(x_{1}\right) \\
& \quad-\frac{i l}{U_{c}^{\prime 2} \eta^{2}}\left[U_{c} T_{0_{c}}+\sigma U_{c}^{\prime} T_{0_{c}} \eta+\sigma U_{c} T_{0_{c}}^{\prime} \eta-\frac{\sigma T_{0_{c}} U_{c}^{\prime \prime} \eta}{\alpha}\right] \mathcal{U}^{\prime}\left(x_{1}\right) \\
& \quad+\frac{\sigma l T_{0}}{U_{c}^{\prime} \eta}\left\{i Q_{ \pm} \mathcal{C}\left(x_{1}\right)-\mathscr{D}_{ \pm}\left(x_{1}\right)\right\} .
\end{aligned}
$$

where $\mathscr{B}_{ \pm}$and $Q_{ \pm}=$const. are defined by $(23 a, b)$, respectively. Of course, in view of these equations, the terms in the braces are independent of the label $( \pm)$.

Note that it is possible to give the corresponding results for the cross-stream velocity component, and for the perturbation density and temperature. Since these results are not needed for the derivation of the amplitude equation, we shall omit them for brevity. Observe that $u$ and $w$ in the main layers are more singular as $\eta \rightarrow 0$ (i.e. $\left.y \rightarrow y_{c}\right)$ for oblique modes $(l \neq 0)$ than for two-dimensional modes $(l=0)$. This is really the main reason why the amplitude scalings (i.e. $\epsilon=\epsilon(\sigma))$ are different for the corresponding nonlinear problems. 


\section{Appendix B. Quadratic nonlinearities}

The equations that govern the evolution of the quadratic nonlinearities (i.e. first and zeroth harmonics) in the critical layer are

$$
\begin{gathered}
\mathscr{L}^{(0)} \hat{u}^{(1 / 2)}+U_{c}^{\prime} \hat{v}^{(1 / 2)}=-N^{(0)} \hat{u}^{(0)} . \\
\mathscr{L}^{(0)} \hat{w}^{(1 / 2)}=-N^{(0)} \hat{w}^{(0)} \\
\frac{\partial \hat{u}^{(1 / 2)}}{\partial \xi}+\frac{\partial \hat{v}^{(1 / 2)}}{\partial \eta}+\ell \frac{\partial \hat{w}^{(1 / 2)}}{\partial \zeta}=0
\end{gathered}
$$

where $\mathscr{L}^{(0)}$ and $N^{(0)}$ are defined by $(19 b)$ and $(35 b)$, respectively. These equations may be obtained from (1), (34), and (35). Because the critical layer is a thin layer, $\partial \hat{p}^{(1 / 2)} / \partial \eta$ $=0$; therefore, without loss of generality, we have set $\hat{p}^{(1 / 2)}=0$. Thus, the outer and main expansions contain no terms of the form $\left[\sigma^{1 / 2}(\cdot)^{(1 / 2)}\right]$.

Next, we multiply out the nonlinear forcing terms on the right-hand sides of (B $1 a, b)$ and write them in Fourier-series representation:

$$
\left.\left.\begin{array}{l}
N^{(0)} \hat{u}^{(0)} \\
N^{(0)} \hat{w}^{(0)}
\end{array}\right\}=\sum_{n=-2}^{2} \sum_{m=-2}^{2} \begin{array}{l}
\mathscr{F}_{\mathrm{n}, \mathrm{m}}^{(1 / 2)} \\
\mathscr{K}_{\mathrm{n}, \mathrm{m}}^{(1 / 2)}
\end{array}\right\} \exp [i(n \xi+m \xi)] .
$$

where the $\mathscr{F}^{\prime} s$ and $\mathscr{K}^{\prime} s$ are the complex Fourier coefficients. Direct calculation shows that $\mathscr{K}_{0,0}^{(1 / 2)}=\mathscr{K}_{2,0}^{(1 / 2)}=0$; these, in view of $\left(\mathrm{B}(b)\right.$ and null initial conditions at $x_{1} \rightarrow-\infty$, immediately imply $(40 a)$.

We recall that the lowest order critical-layer solutions (e.g. (39)) are linear in the vortex sheet parameter $\Lambda$ and, setting $\Lambda=0$, we recover the corresponding critical-layer solutions given by Goldstein \& Lee (1992). Thus, for the sake of brevity, we write

$$
\mathscr{F}_{0,0}^{(1 / 2)}=(\mathrm{GL})-\frac{\Lambda \ell^{2} \alpha T_{0_{c}}}{8 i}\left[W_{0}+\eta \frac{\partial W_{0}}{\partial \eta}\right] \tilde{\mathcal{L}}-\frac{\Lambda \ell^{2} T_{0_{c}}}{8} \tilde{\mathcal{L}} \cdot \frac{\partial W_{0}}{\partial \eta}+\text { c.c. }
$$




$$
\begin{aligned}
& \mathscr{F}_{0.2}^{(1 / 2)}=(\mathrm{GL})+\frac{\Lambda \ell^{2} \alpha T_{0_{c}}}{16 i} \tilde{\mathcal{L}}\left[W_{0}-\eta \frac{\partial W_{0}}{\partial \eta}\right]-\frac{\Lambda \ell^{2} T_{0_{c}}}{16} \tilde{\mathcal{L}} \cdot \frac{\partial W_{0}}{\partial \eta}+\text { c.c. } \\
& F_{2.0}^{(1 / 2)}=(\mathrm{GL})+\frac{i U_{c}^{\prime}{ }^{2} \Lambda^{2}}{8} \mathscr{C}^{2}-\frac{\Lambda l^{2} T_{0_{c}}}{8} \mathcal{L}^{\prime} \frac{\partial W_{0}}{\partial \eta} \\
& -\frac{\Lambda \ell^{2} \alpha T_{0}}{8 i}\left[3 W_{0}-\eta \frac{\partial W_{0}}{\partial \eta}\right] \mathcal{L} \\
& \mathscr{K}_{0.2}^{(1 / 2)}=(\mathrm{GL})+\frac{\Lambda \ell \alpha T_{0_{c}}}{16 i}\left[W_{0}+\eta \frac{\partial W_{0}}{\partial \eta}\right] \tilde{\mathcal{L}}^{\prime}+\frac{\Lambda \ell T_{0_{c}}}{16} \tilde{\mathcal{A}}^{\prime} \frac{\partial W_{0}}{\partial \eta}-\text { c.c. }
\end{aligned}
$$

where $(G L)$ denotes the terms that are available, at least in principle (see (5.11), (5.13), (5.25), and (5.26) in Goldstein \& Lee (1992)). Of course, the notation is very different, so it is not easy to make a symbol-by-symbol comparison; nevertheless, even a quick glance reveals that the solutions are equivalent because they contain the same basic information. Note also that c.c. refers to the complex conjugate of the terms that have $\Lambda$ as a factor in them.

After using (B 3) and (B 2) in the governing equations. (B 1). and solving these for the Fourier coefficients of the velocity components, we find

$$
\begin{aligned}
& \hat{W}_{0.2}^{(1 / 2)}=(\mathrm{GL})+\frac{\Lambda \ell \alpha T_{0_{c}}}{16 i U_{\mathrm{c}}}-\tilde{\ell} W_{1}-\text { c.c. }, \\
& \hat{V}_{0.2}^{(1 / 2)}=(\mathrm{GL})+\frac{i T_{0_{c}} \ell^{2} \Lambda}{8 U_{c}}\left(\tilde{\mathcal{L}} W_{0}-\mathcal{L} \tilde{W}_{0}\right) \\
& \hat{V}_{2.0}^{(1 / 2)}=(\mathrm{GL})-\frac{i T_{0_{c}} \ell^{2} \Lambda}{4 U_{c}}-\mathcal{L} W_{0}-\frac{i U_{c}{ }_{c} \Lambda^{2}}{8} \mathcal{L}^{2} . \\
& \hat{U}_{0,0}^{(1 / 2)}=(\mathrm{GL})+\frac{i T_{0} \alpha \ell^{2} \Lambda}{8 U_{\mathrm{c}}} \tilde{\mathcal{L}} W_{1}+\text { c.c. } \\
& \hat{U}_{0.2}^{(1 / 2)}=(\mathrm{GL})+\frac{T_{0_{c}} \alpha \ell^{2} \Lambda}{16 U_{c}} \tilde{\mathcal{L}} W_{1}+\text { c.c. } \\
& \hat{U}_{2,0}^{(1 / 2)}=(\mathrm{GL})+\frac{i T_{0} \alpha \ell^{2} \Lambda}{8 U_{\mathrm{c}}} \mathcal{A} W_{1}
\end{aligned}
$$


Recall that $W_{n}\left(\eta, x_{1}\right)=W_{n}(n=0,1,2 \ldots)$ are defined by $(24 b)$ and behave as $\eta-(1+n)$ for $\eta \rightarrow \pm \infty$. Thus, it is possible to match the entire critical-layer solution to that in the main layers as given by the linearized theory of $\$ 4$. Of course, this asymptotic matching is carried out only to the order of accuracy of the analysis. Also note that when the expressions in $(41 b-h)$ are multiplied out using (39c) and (B 4), $T_{\mathrm{k}}(k-1,2, \ldots$ 7) become polynomials of degree no greater than two in the vortex sheet displacement parameter, $\Lambda$. The evolution equation for the amplitude is expected to depend only on even powers of $\Lambda$, since the mirror image of the wavy wall, $(6 g)$, cannot change the stability characteristics of the oblique modes. Details may be found in Gartside (1995).

Our final remark concerns the validity of $(40 b)$. This result follows immediately from (B IC) under the observation that $\partial \hat{V}_{2,2}^{(1 / 2)} / \partial \eta=0$. This comes from the initial conditions at $x_{1} \rightarrow-\infty$ and asymptotic matching. (Note that $\hat{V}_{2.2}^{(1 / 2)}$ can be shown to satisfy $\left(\partial / \partial x_{1}+2 i \alpha \eta\right)\left(\partial^{2} \hat{V}_{2,2}^{(1 / 2)} / \partial \eta^{2}\right)=0 ;$ we omit the details, however. $)$

\section{Appendix C. An illustrative calculation for the phase jump arising from the} nonlinear terms

Let us consider the contribution of the nonlinear forcing term. $T_{7}$, to the phasejump equation. After using (B $4 a)$ in $(41 h)$ and solving $(37 c)$ for $Q_{1,1}^{(7)}$, we obtain

$$
\begin{gathered}
Q_{1,1}^{(7)}\left(\eta, x_{1}\right)=\frac{i \Lambda^{2} \ell^{2} \alpha^{3} T_{0_{c}}}{32 U_{c}} \int_{-\infty}^{x_{1}} \mathcal{K}^{2}\left(\hat{x}_{1}\right) \tilde{I}_{2}\left(\eta, \hat{x}_{1}\right) \exp \left[i \alpha \eta\left(2 \hat{x}_{1}-x_{1}\right)\right] \mathrm{d} \hat{x}_{1} \\
-\frac{i \Lambda^{2} \ell^{2} \alpha^{3} T_{0_{c}}}{32 U_{c}} \exp \left(-i \alpha \eta x_{1}\right) \int_{-\infty}^{x_{1}}\left|\mathcal{L}\left(\hat{x}_{1}\right)\right|^{2} I_{2}\left(\eta, \hat{x}_{1}\right) \mathrm{d} \hat{x}_{1} \\
+\Lambda(\ldots) .
\end{gathered}
$$

where, for the purposes of illustration, we consider only the first two terms in (C I). These are proportional to $\Lambda^{2}$. Recall that $I_{\mathrm{n}}\left(\eta, x_{1}\right)(n=0,1, \ldots)$ are defined by $(24 c)$ and $Q_{1,1}^{(7)}$ is the contribution of $T_{7}$ to $Q_{1,1}$. 
In order to evaluate

$$
\int_{-\infty}^{\infty} Q_{1,1}^{(7)}\left(\eta, x_{1}\right) \mathrm{d} \eta
$$

we substitute the definition of $I_{2}$ into $(C 1)$ and use the well-known result

$$
2 \pi \delta(x)=\int_{-\infty}^{\infty} \exp (i x \eta) \mathrm{d} \eta
$$

where $\delta(x)$ is the Dirac delta function. The triple integral in $(C 2)$ is thus reduced to a double integral; the latter is further reduced to a single integral by the substitution property of the delta function. The final result is

$$
\begin{aligned}
\int_{-\infty}^{\infty} Q_{1,1}^{(7)}\left(\eta, x_{1}\right) \mathrm{d} \eta= & \frac{i \Lambda^{2} \ell^{2} \alpha^{3} T_{0}}{32 U_{c}} \frac{2 \pi}{|\alpha|} \int_{-\infty}^{x_{1}}\left(\hat{x}_{1}-x_{1}\right)^{2} \mathcal{L}^{2}\left(\hat{x}_{1}\right) \tilde{\mathcal{L}}\left(2 \hat{x}_{1}-x_{1}\right) \mathrm{d} \hat{x}_{1} \\
& +\Lambda(\ldots)
\end{aligned}
$$

to which the second term on the right-hand side of $(\mathrm{C}$ 1) has actually made a null contribution. We emphasize that this single integral represents a new term that has not appeared previously in the literature for the oblique-mode self-interaction problem.

\section{REFERENCES}

ARtolA, M. \& MAJDA, A. 1987 Nonlinear development of instabilities in supersonic vortex sheets. The basic kink modes. Physica D 28. 253-281.

BAlsA, T. F. \& GoldsteIN, M. E. 1990 On the instabilities of supersonic mixing layers: a high-Mach-number asymptotic theory. J. Fluid Mech. 216, 585-611.

BENNEY, D. J. \& BERGERON, R. F. 1969 A new class of nonlinear waves in parallel flows. Stud. Appl. Math. 48, 181-204. 
DRAZIN, P. G. \& REID. W. H. 1981 Hydrodynamic Stability. Cambridge University Press.

FEJER. J. \& MILES, J. 1963 On the stability of a plane vortex sheet with respect to three-dimensional disturbances. J. Fluid Mech. 15, 335-336.

GAJJAR, J. S. B. 1993 Nonlinear evolution of the first mode supersonic oblique waves in compressible boundary layers. Part I. Heated/cooled walls. NASA TM 106087.

GARTSIDE. J. 1995 Nonlinear interaction of two oblique modes in a supersonic mixing layer. Ph.D. dissertation, Program in Applied Mathematics, Univ. Arizona, Tucson.

Goldstein, M. E. \& CHOI. S. W. 1989 Nonlinear evolution of interacting oblique waves on two-dimensional shear layers. J. Fluid Mech. 207, 97-120. Also, corrigendum, $J$. Fluid Mech. 216, 659-663.

Goldstein, M. E. DuRbin. P. \& Leib, S. 1987 Roll-up of vorticity in adversepressure-gradient boundary layers. J. Fluid Mech. 183, 325-342.

Goldstein, M. E. \& LEE, S. S. 1992 Fully coupled resonant-triad interaction in an adverse-pressure-gradient boundary layer. J. Fluid Mech. 245, 523-551.

GoldsteIN, M. E. \& LEIB. S. 1989 Nonlinear evolution of oblique waves on compressible shear layers. J. Fluid Mech. 207, 73-96.

HiCKERNELl, F. J. 1984 Time-dependent critical layers in shear flows on the betaplane. J. Fluid Mech. 142, 431-449.

HUERRE, P. \& MONKEwItz, P. A. 1990 Local and global instabilities in spatially developing flows. Ann. Rev. Fluid Mech. 22, 473-537.

JACKSON, T. L. \& GROSCH, C. E. 1989 Inviscid spatial stability of a compressible mixing layer. J. Fluid Mech. 208, 609-637.

LEES, L. \& LIN, C. C. 1946 Investigation of the stability of the laminar boundary layer in a compressible fluid. NACA TN-1115. 
LighthILl, M. J. 1978 Fourier Analysis and Generalized Functions. Cambridge University Press.

MASLowe, S. A. 1981 Shear flow instabilities and transition. In Hydrodynamic Instabilities and the Transition to Turbulence (ed. H. L. Swinney \& J. P. Gollub). pp. 181-228. Springer-Verlag.

MILES, J. 1958 On the disturbed motion of a plane vortex sheet. J. Fluid Mech. 4, $538-552$.

STEWARTSON. K. 1981 Marginally stable inviscid flows with critical layers. IMA J. Appl. Math. 27, 133-175.

WU, X., LEE. S.-S. \& COWLEY. S. 1993 On the weakly nonlinear three-dimensional instability of shear layers to pairs of oblique waves: the Stokes layer as a paradigm. J. Fluid Mech. 253, 681-721. 


\section{LIST OF FIGURES}

Figure 1. Geometry of mixing layer and instability mode (schematic).

Figure 2. Triple-layer structure of supersonic instability modes at low frequency. $\omega=O(\sigma) \ll 1$.

Figure 3. Real part of similarity parameter, $K$, as a function of Mach number, $m$, for three temperature ratios. $\theta=30^{\circ}$, fast mode.

Figure 4. Real part of similarity parameter. $K$, as a function of Mach number, $m$, for three temperature ratios. $\theta=30^{\circ}$, slow mode.

Figure 5. Universal behavior of magnitude of amplitude as a function of streamwise coordinate $x_{1}, \theta=30^{\circ}$.

Figure 6. Linear variation of phase $(A)$ as a function of streamwise coordinate $x_{1}$. $\theta=$ $30^{0}, T_{0_{-}}=2$, slow mode: $(\longrightarrow) m=5 ;(--) m=6 ;(\cdots) m=7$.

Figure 7. Universal behavior of magnitude of amplitude as a function of streamwise coordinate $x_{1}, \theta=60^{\circ}$.

Figure 8. Stepwise-linear variation of phase $(A)$ as a function of streamwise coordinate $x_{1} . \theta=60^{\circ}, T_{0_{-}}=1.0$, fast mode: $(-) m=7.5 ;(-) m=8.5 ;(\cdots) m=$ 9.5. 


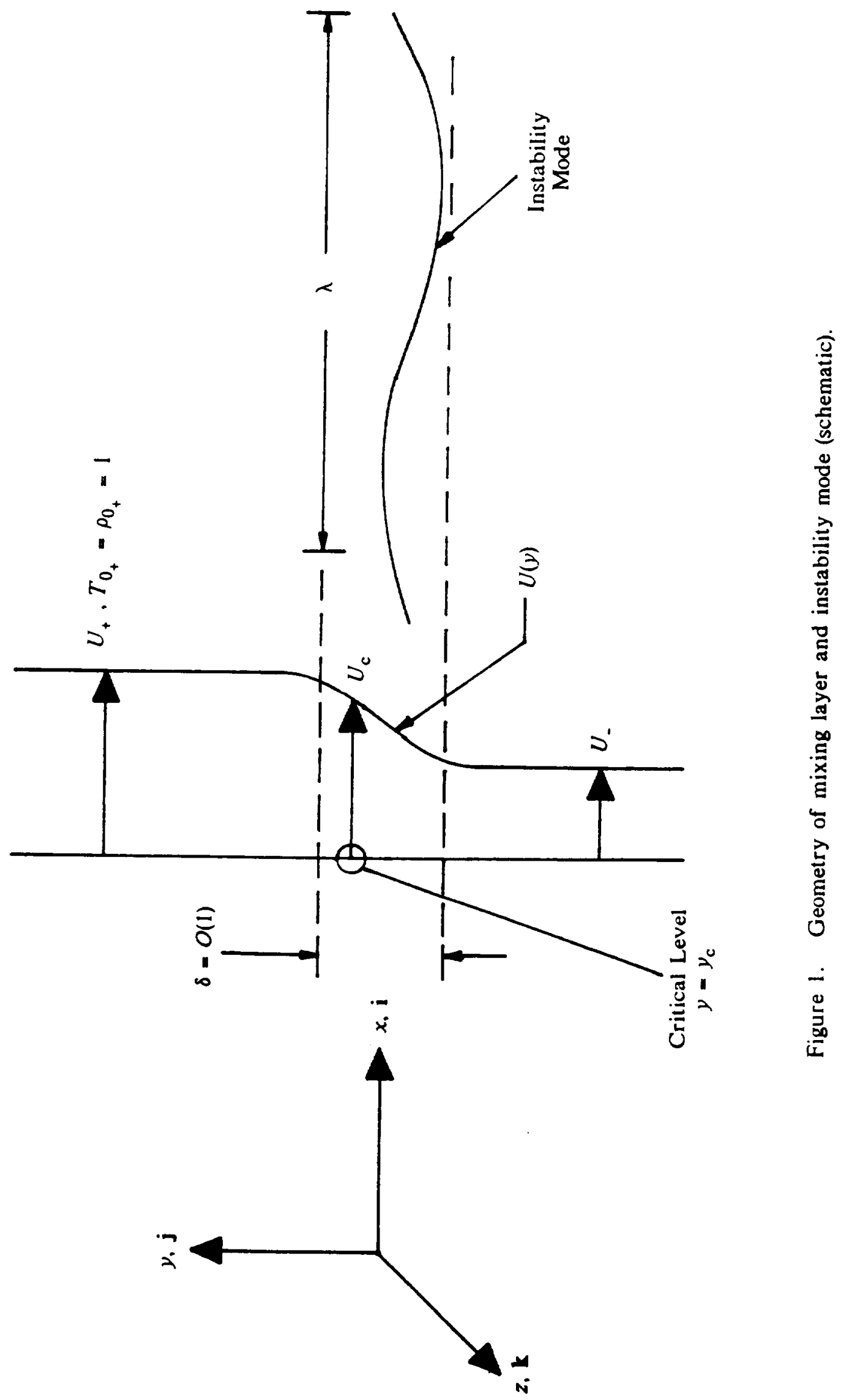


$\frac{1}{b}=1$

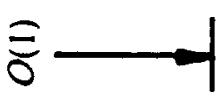

$\frac{0}{\sigma} \longrightarrow 1+\frac{\sigma}{0}$

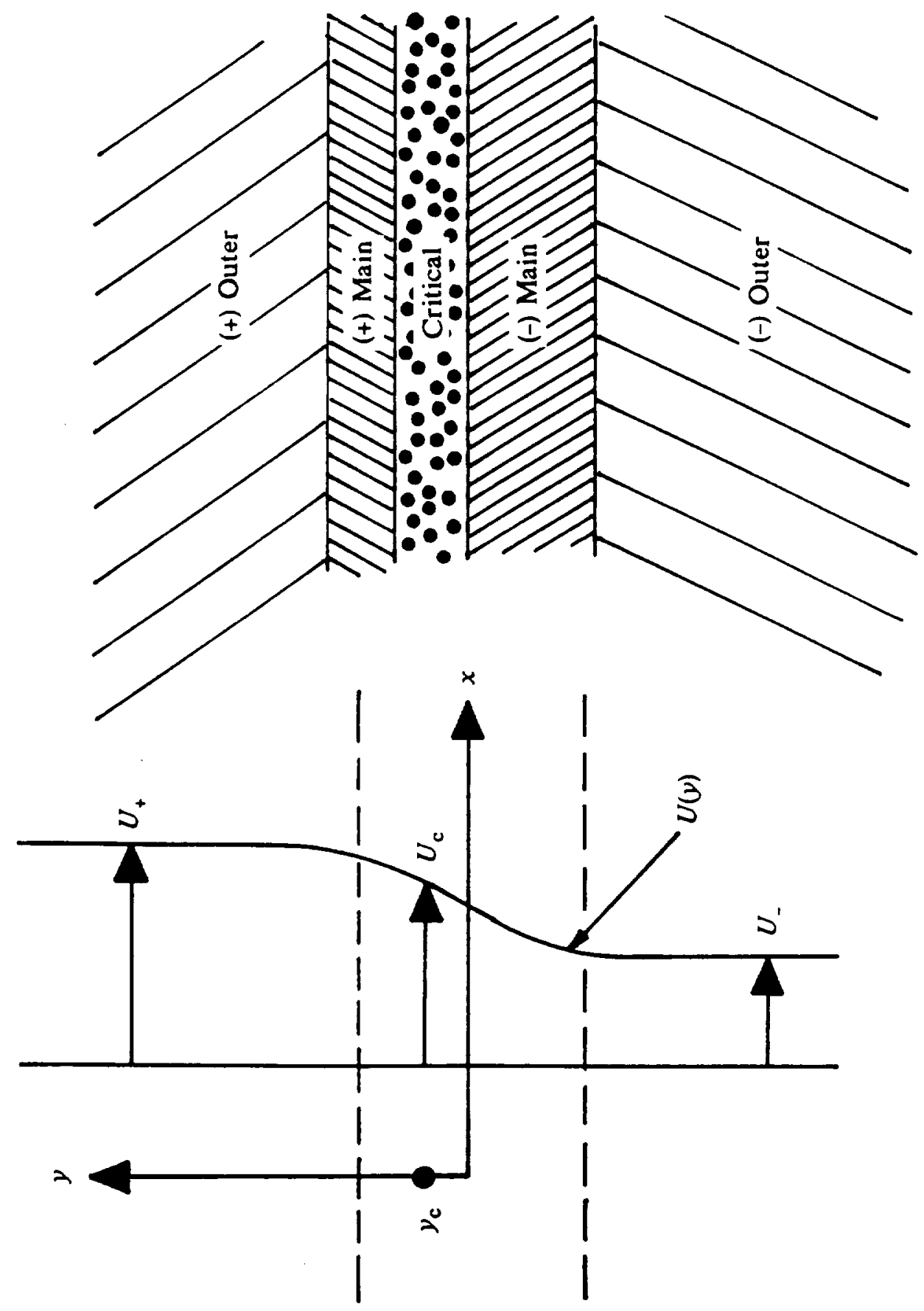




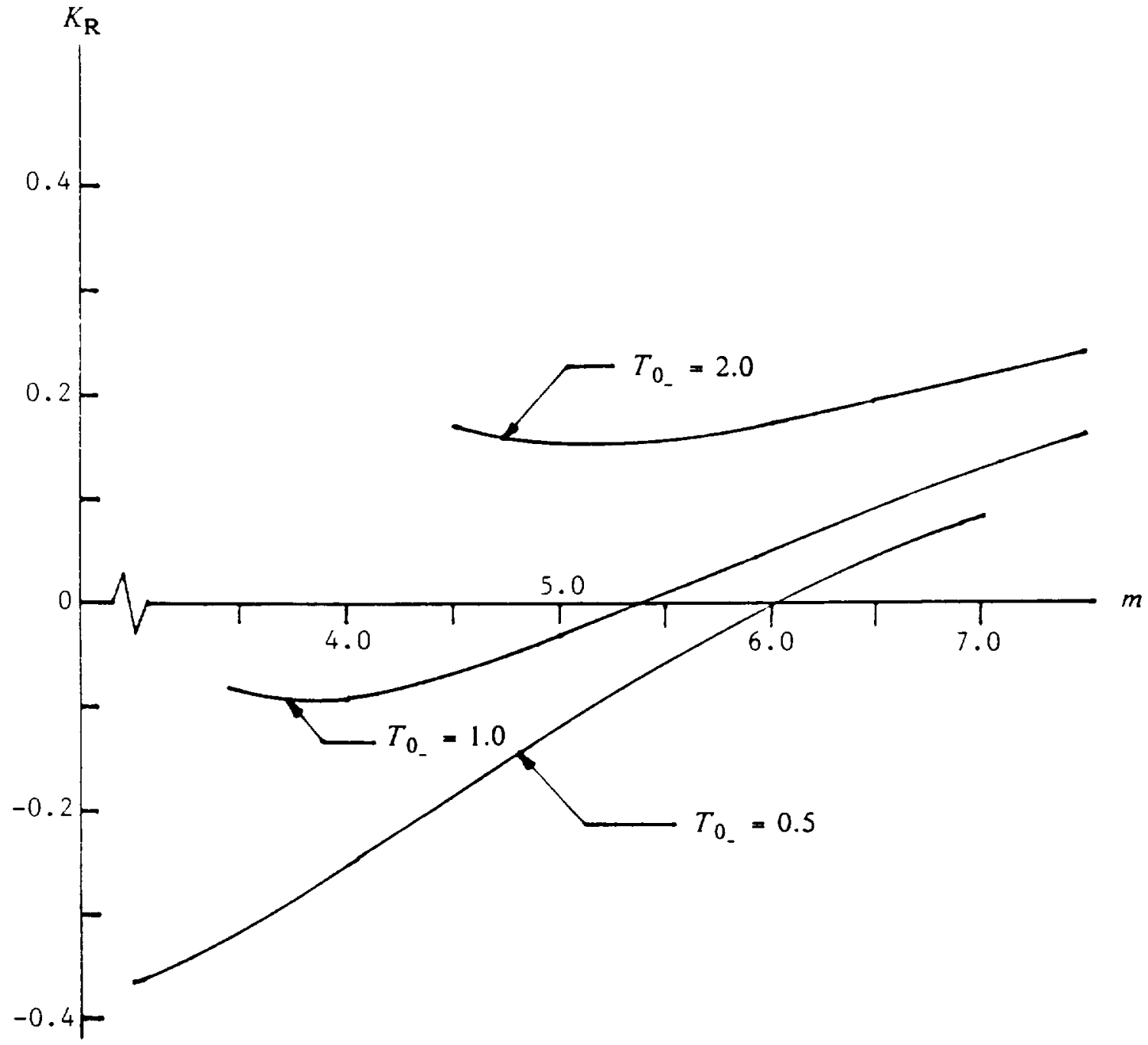

Figure 3. Real part of similarity parameter, $K$, as a function of Mach number, $m$, for three temperature ratios. $\theta=30^{\circ}$, fast mode. 


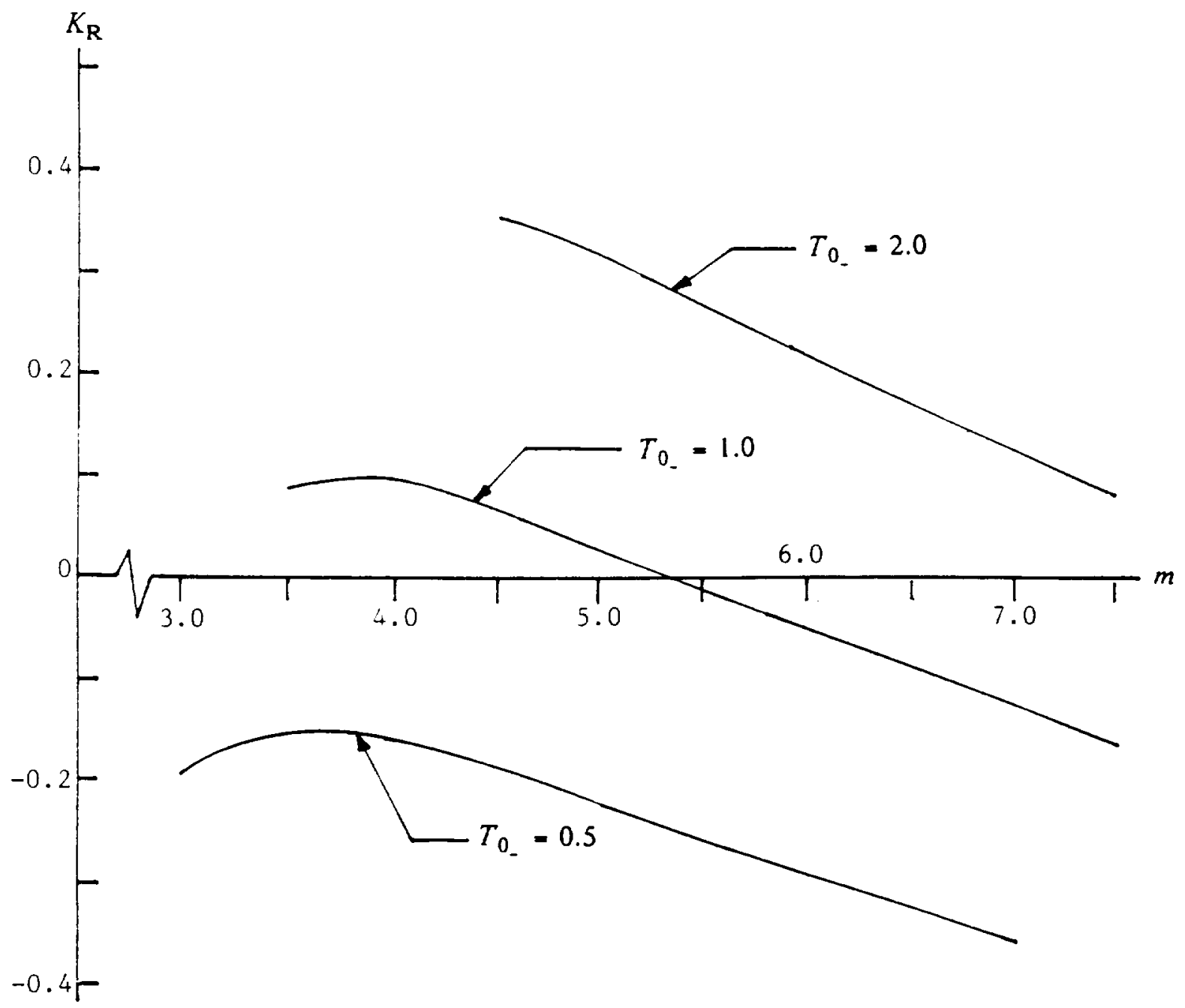

Figure 4. Real part of similarity parameter, $K$, as a function of Mach number, $m$, for three temperature ratios. $\theta=30^{\circ}$, slow mode. 


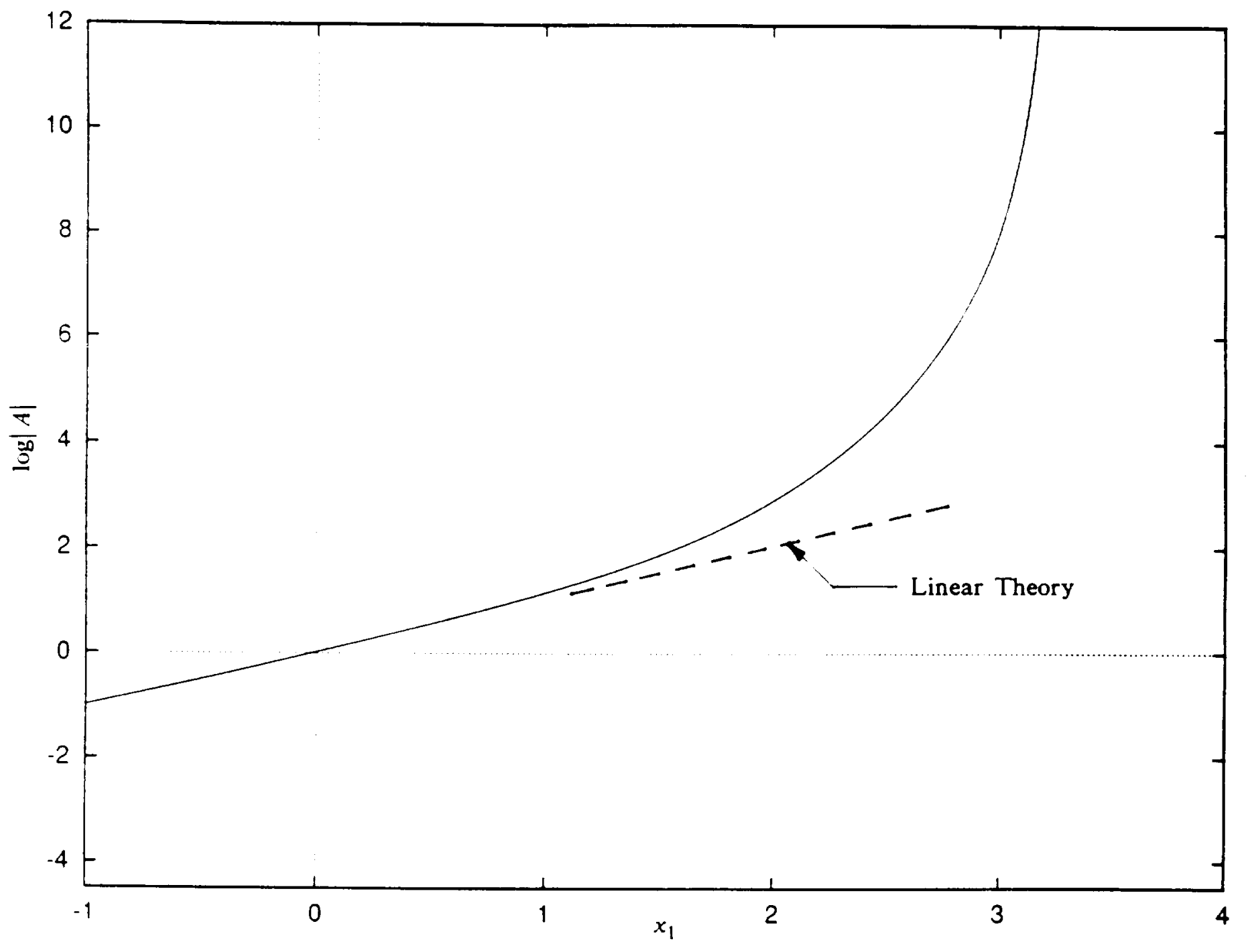

Figure 5. Universal behavior of magnitude of amplitude as a function of streamwise coordinate $x_{1}, \theta=30^{\circ}$. 


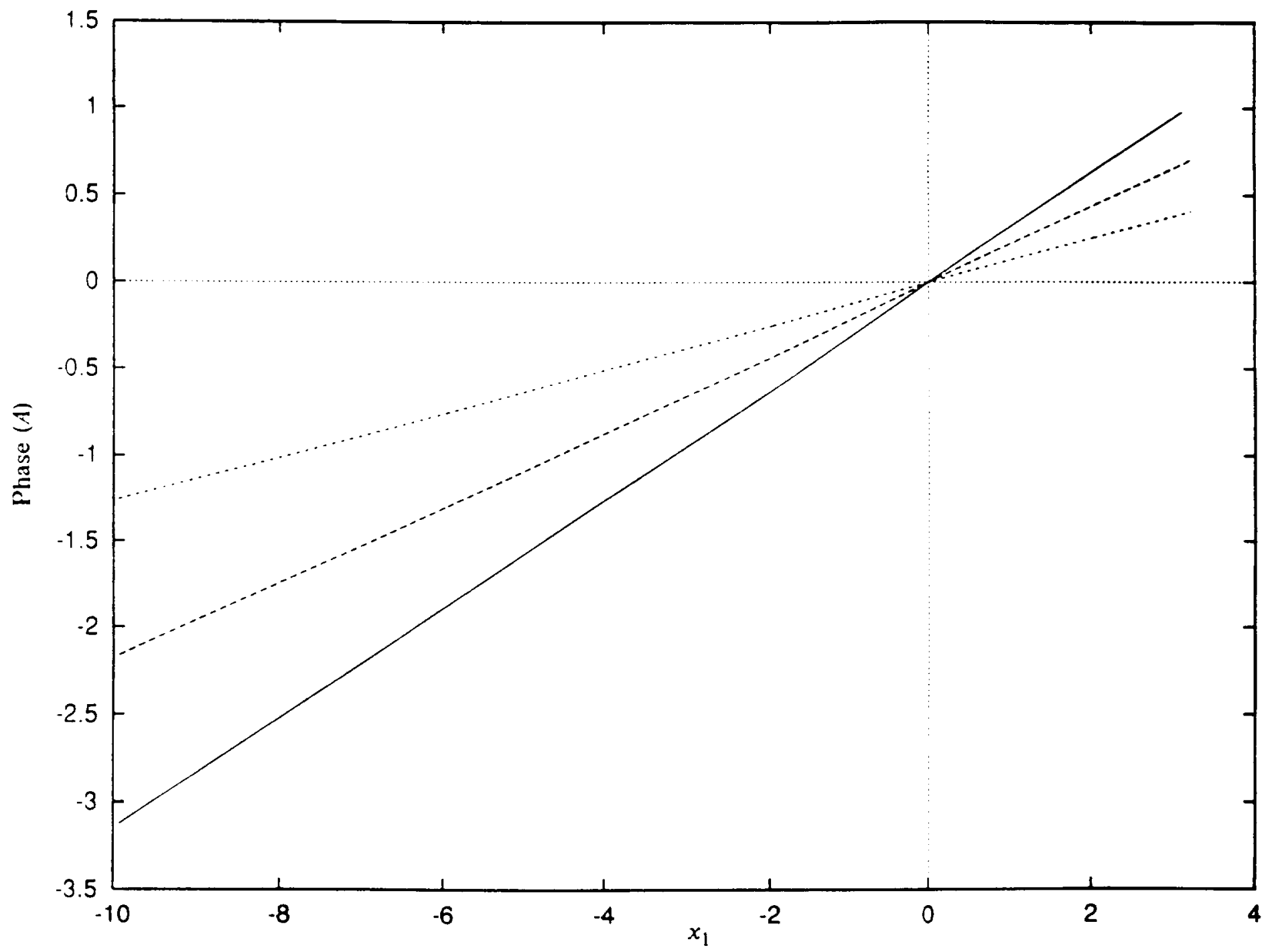

Figure 6. Linear variation of phase $(A)$ as a function of streamwise coordinate $x_{1}$. $\theta=$ $30^{0}, T_{0_{-}}=2$, slow mode: $(\longrightarrow) m=5 ;(--) m=6 ;(\cdots) m=7$. 


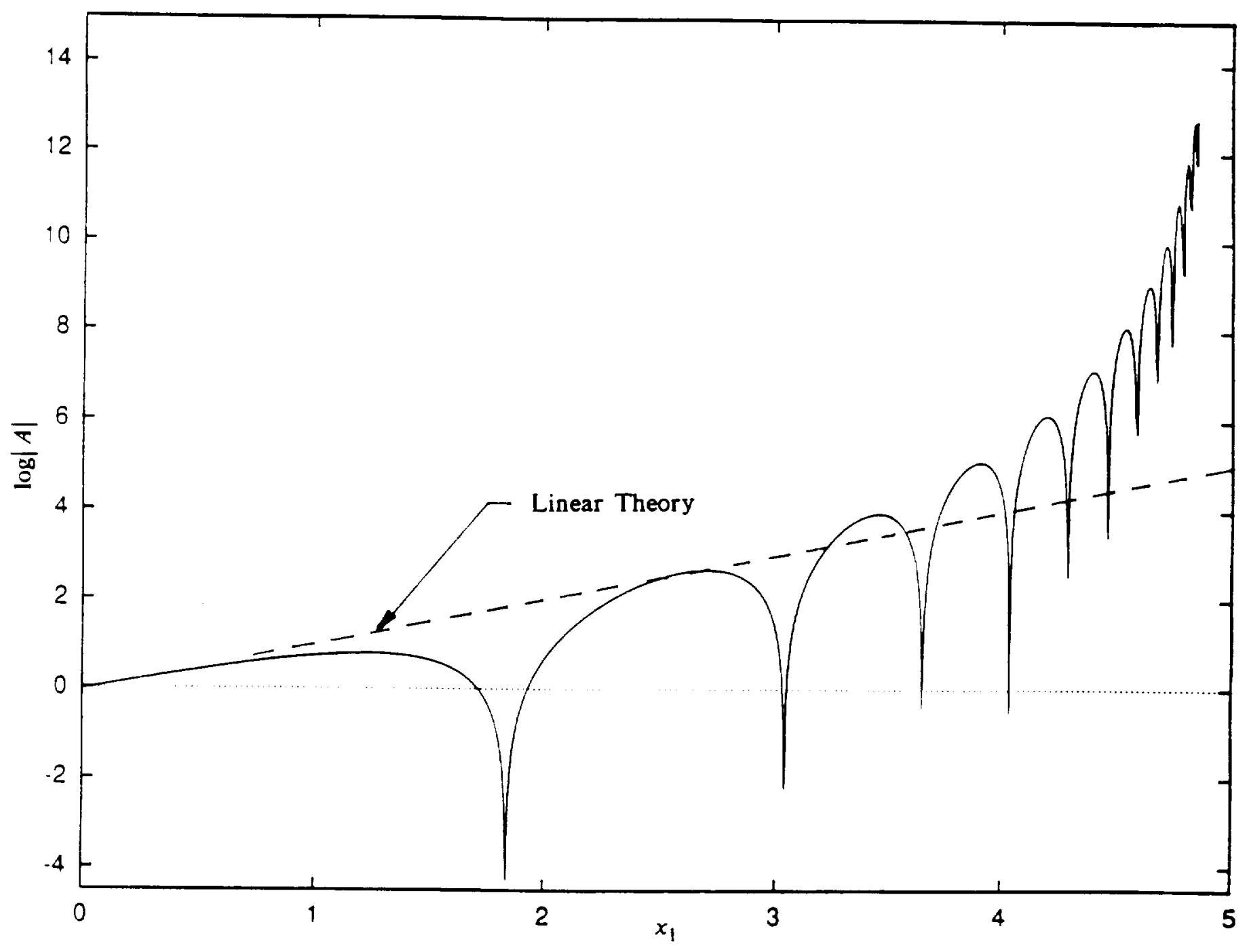

Figure 7. Universal behavior of magnitude of amplitude as a function of streamwise coordinate $x_{1} . \theta=60^{\circ}$. 


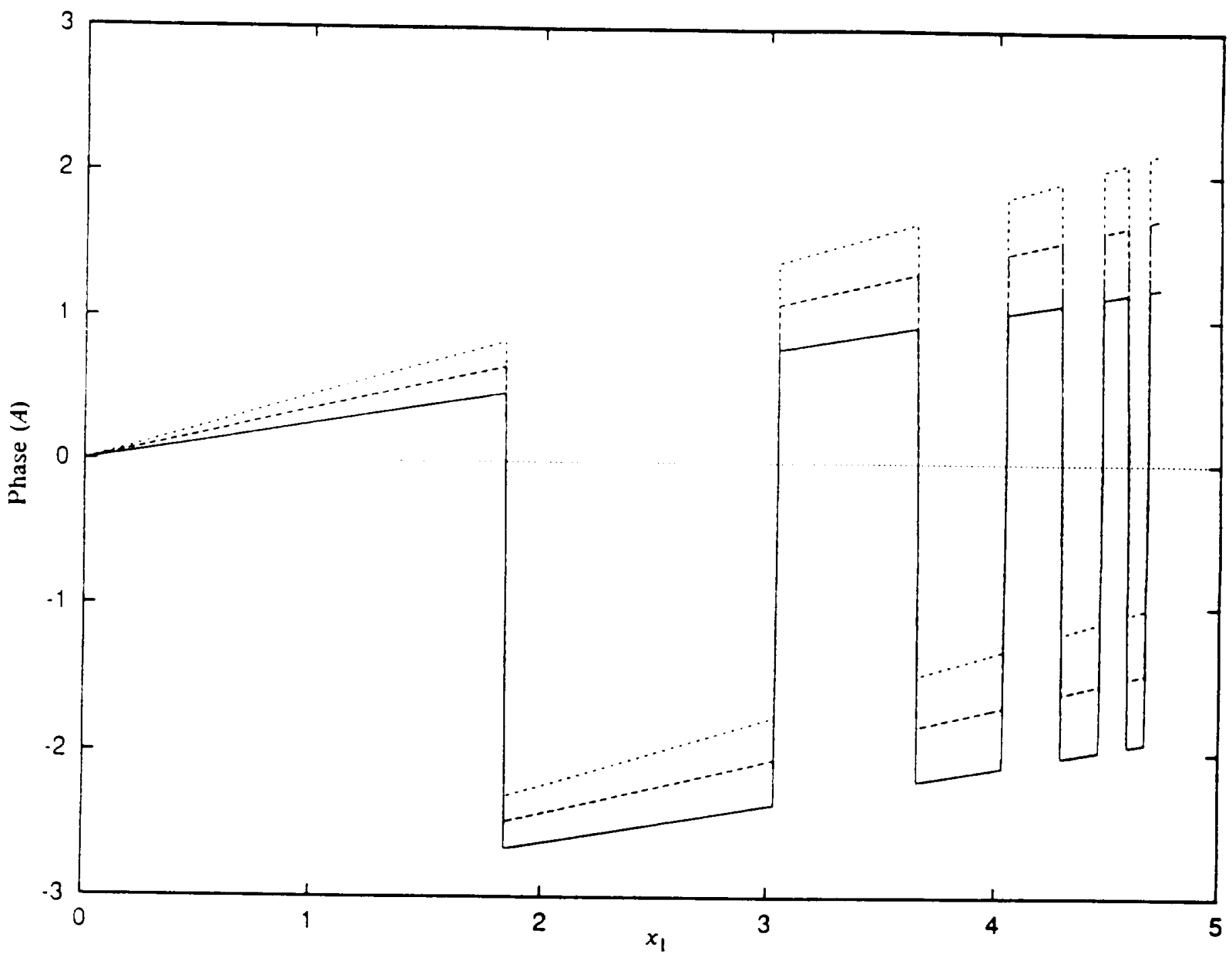

Figure 8. Stepwise-linear variation of phase $(A)$ as a function of streamwise coordinate $x_{1} . \theta=60^{\circ}, T_{0_{-}}=1.0$. fast mode: $(\longrightarrow m=7.5 ;(-) m=8.5 ;(\cdots) m=$ 9.5. 